\title{
Practical issues related to uterine pathology: staging, frozen section, artifacts, and Lynch syndrome
}

\author{
Robert A Soslow \\ Department of Pathology, Memorial Sloan Kettering Cancer Center, New York, USA
}

\begin{abstract}
This review covers three areas in endometrial tumor pathology: International Federation of Gynecology and Obstetrics (FIGO) staging, the use of frozen section, and Lynch syndrome. The section on FIGO staging will emphasize problems that practicing pathologists often confront, such as measuring the depth of myometrial invasion, assessing for the presence of cervical stromal invasion, detecting low-volume lymph node metastases, and recognizing synchronous endometrial and ovarian tumors and artifacts. The frozen section portion of this review will focus on the performance characteristics of intraoperative examination of the uterus to determine tumor grade and depth of myometrial invasion, including suggestions for alternative methods. The last portion of this review will provide an overview of Lynch syndrome and a discussion of the rationale and methods of screening for Lynch syndrome.
\end{abstract}

Modern Pathology (2016) 29, S59-S77; doi:10.1038/modpathol.2015.127

\section{FIGO staging}

Table 1 provides the 2009 International Federation of Gynecology and Obstetrics (FIGO) staging formulation for endometrial carcinoma and carcinosarcoma, ${ }^{1}$ which is simplified from the 1988 classification. Pathologists no longer need to distinguish less than $50 \%$ myometrial invasion from no myometrial invasion; use peritoneal washing status to inform stage; or differentiate between endocervical mucosal and endometrial involvement by tumor. Despite these simplifications, many complexities and unanswered questions remain. FIGO staging ignores significant clinical heterogeneity within each substage. For example, a 70-year-old patient with serous carcinoma who has undergone a hysterectomy, without peritoneal or lymph node staging and with $40 \%$ myometrial invasion (FIGO stage I), has an estimated 5-year survival rate of $66 \%$; the same patient with FIGO grade 1 endometrioid carcinoma, 30 negative lymph nodes, and no myometrial invasion (FIGO stage I) has an estimated 5-year survival rate of 97\% (http://nomo grams.mskcc.org/Uterine/EndometrialSurvival.aspx).

Correspondence: Dr RA Soslow, MD, Department of Pathology, Memorial Sloan Kettering Cancer Center, 1275 York Avenue, New York, NY 10065, USA.

E-mail: soslowr@mskcc.org

Received 16 July 2015; revised 18 September 2015; accepted 26 September 2015
Given this example, it becomes apparent that the current FIGO staging system does not take into account the distinctiveness of each type of endometrial carcinoma or staging adequacy. We recently reported a proposal for substaging uterus-confined endometrial carcinoma that recognizes disease type and grade, as well as staging adequacy. ${ }^{2}$ This yields significantly more predictive information than does either the FIGO 1988 or FIGO 2009 systems, with uterus-confined unstaged serous or FIGO grade 3 carcinomas having the worst prognosis and patients with well-staged FIGO grade 1 endometrioid carcinomas having the best outcome. This system also fails to recognize the importance of different modes of dissemination, suggesting that lymphovascular and peritoneal spread, direct extension, and implantation without invasion are clinically equivalent. Furthermore, FIGO 2009 does not indicate whether lymphovascular tumor emboli in ovarian hilar vessels should be considered stage III disease, nor does it define cervical stromal invasion. Several other challenges pathologists face when trying to implement the FIGO 2009 staging system have been discussed. ${ }^{3-5}$

\section{Myometrial Invasion}

Assessment for the presence and depth of myometrial invasion can be difficult. Several studies have reported interpathologist discrepancy rates of $\sim 30 \%$, with gynecological pathologists tending to report smaller measurements than non-specialized 
Table 1 International Federation of Gynecology and Obstetrics (FIGO) 2009 staging of endometrial carcinoma and carcinosarcoma

IA: Tumor confined to the uterus; no or $<1 / 2$ myometrial invasion

IB: Tumor confined to the uterus; $1 \frac{1}{2}$ or more myometrial invasion

II: Cervical stromal invasion, but not beyond uterus

IIIA: Tumor invades serosa or adnexa

IIIB: Vaginal and/or parametrial involvement

IIIC1: Pelvic node involvement

IIIC2: Para-aortic involvement

IVA: Tumor invasion bladder and/or bowel mucosa

IVB: Distant metastases including abdominal metastases and/ or inguinal lymph nodes

pathologists. ${ }^{6-9}$ We have found that overcalling the presence and depth of myometrial invasion can be attributed to irregular endomyometrial junction, inapparent and metaplastic endometrial stroma, and measuring tumor thickness rather than depth. To reiterate, the location of the endomyometrial junction must be ascertained or at least estimated to avoid measuring tumor thickness, particularly when the tumor is exophytic. Even if the tumor is massive and protrudes into the endometrial cavity, this does not justify measuring its thickness and reporting that measurement as the depth of myometrial invasion (Figure 1). The less commonly encountered undercalls are related to unfamiliarity with less common invasion patterns: 'microcystic elongated and fragmented' (MELF) and 'adenoma malignum-like.' Figure 2 illustrates the methodology for measuring depth of myometrial invasion. One must take into account the fact that most uteri have an irregular endomyometrial junction. This interface can be assessed at low power and recognized as intact when the contour is rounded, there is endometrial stroma at the interface with myometrium, and a stromal response to invasion is lacking. The same rules apply to the distinction of adenomyosis colonized by adenocarcinoma from myometrial-invasive adenocarcinoma. A particular problem involves the appearance of altered endometrial stroma, which may be composed of spindly eosinophilic cells that resemble myometrium. Failure to recognize this type of stroma might lead to an erroneous diagnosis of myometrial invasion. Examination at high power usually reveals seamless transitions from typicalappearing endometrial stroma and a contrast between the fibrillary appearance of altered stroma and the well-organized bundles of myometrium (Figure 3). Another uncommon problem that is not addressed by the FIGO system is how to categorize a focus of deeply placed myometrial invasion that is discontinuous with the overlying endomyometrial junction and situated close to a focus of adenomyosis colonized by adenocarcinoma (Figure 4). My approach to this scenario is to report the depth of myometrial invasion by measuring the distance between the invasive focus and the adjacent area of adenomyosis. Although this has not been studied

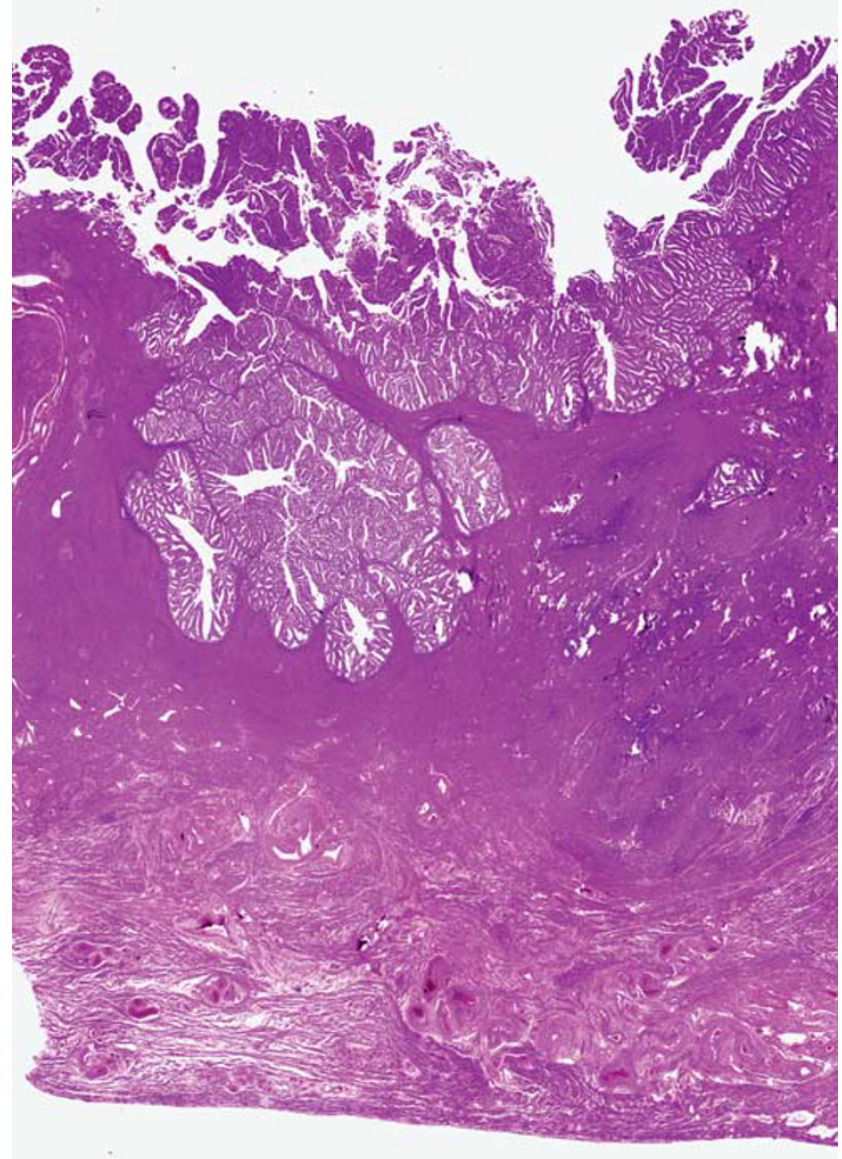

Figure 1 Irregular endomyometrial junction. Rounded contours, focally condensed endometrial stroma at the junction, and the absence of desmoplasia are against the presence of myometrial invasion. (This figure was published in Uterine Pathology, Copyright Cambridge University Press, 2012).

specifically, due to its rarity, it is hard to imagine that 10 cells of myometrial-invasive carcinoma deep in the myometrium has the same clinical implication as a mass of millions of tumor cells arranged contiguously from the overlying endomyometrial junction to the deepest focus.

'MELF' and 'adenoma malignum-like' invasion patterns are not difficult to recognize once a few basic guidelines are considered (Figures 5 and 6). MELF pattern invasion ${ }^{10-17}$ is usually first detected at scanning magnification because of its tendency to elicit an obvious myxoinflammatory myometrial response to invasion. The neoplastic epithelial cells may be difficult to distinguish at first, because they tend to be obscured by the stromal response (Figure 7). The cells that constitute the invasive foci also differ in appearance when compared to adenocarcinoma in the endometrial compartment. They are frequently squamous metaplastic, elongated with attenuated cytoplasm and, paradoxically, may also have a histiocyte-like appearance with a low nuclear-to-cytoplasmic ratio. The cytoplasmic 

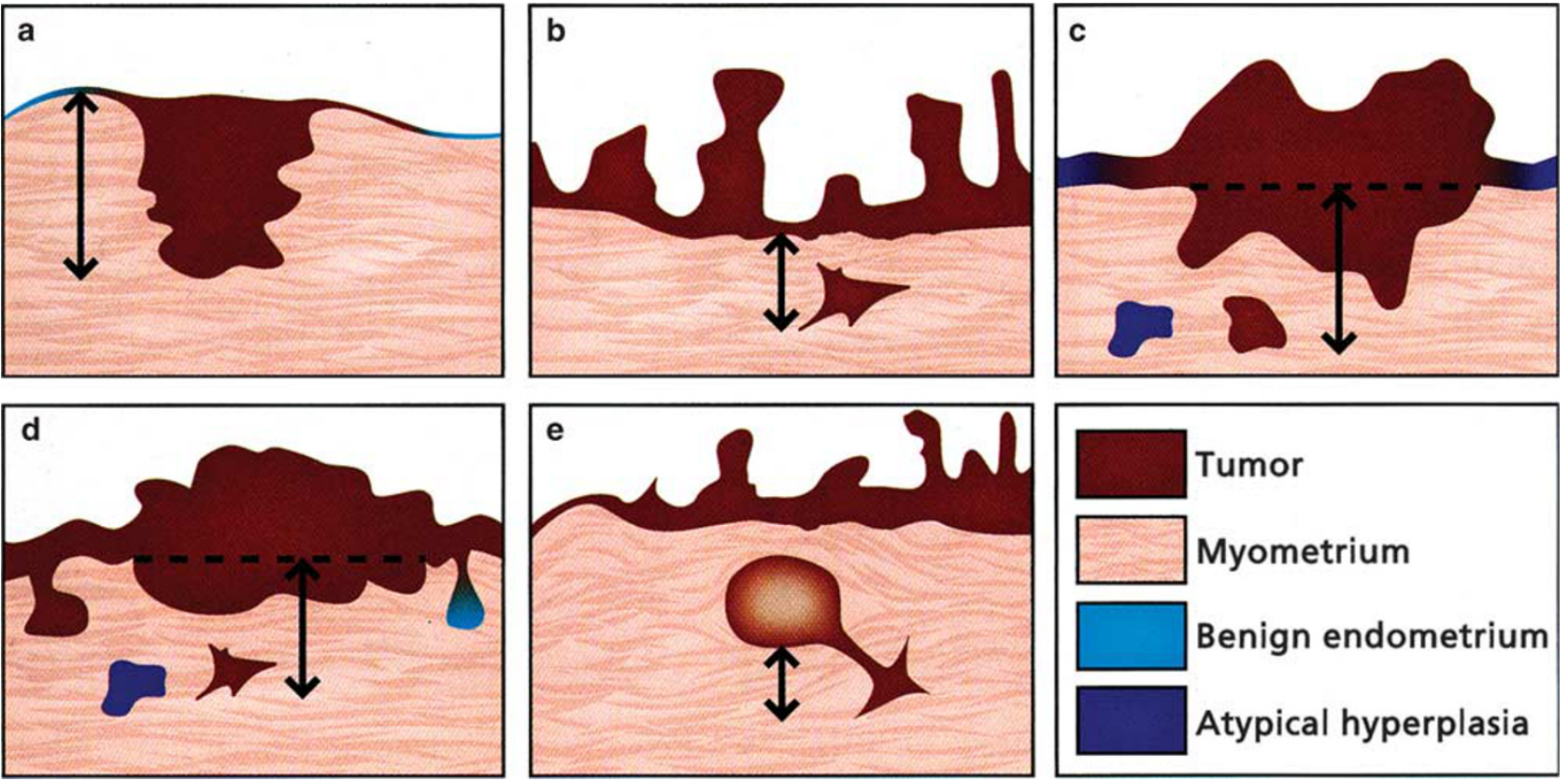

Figure 2 Assessment of myometrial invasion. (a) Direct invasion from the endometrium is the most recognizable and reproducible form of invasion, particularly when the advancing front is jagged and associated with a stromal response. In this situation, the depth of invasion is measured from the nearest adjacent uninvolved endomyometrial junction to the deepest focus of invasion. (b) Discontinuous myometrial invasion; ensure the discontinuous focus is invasive (illustrated by spiculated contours), not adenomyosis colonized by carcinoma (illustrated by rounded contours in $\mathbf{c - e}$ ). (c and $\mathbf{d}$ ) Depth of invasion in these cases is measured from a virtual plane whose location is estimated from the adjacent endomyometrial junction. In $\mathbf{c}$, the invasive focus is represented by a broad, pushing front, a pattern that is difficult to evaluate. Pushing invasion can often be recognized by the presence of a stromal response at the leading edge. In d, the invasive focus is mostly discontinuous; the discontinuous focus of myometrial invasion can be distinguished from adenomyosis because of its spiculated shape. Histologically, the lack of endometrial stroma and the presence of surrounding desmoplasia are the two most helpful features that indicate myometrial invasion is present. (e) Rarely, carcinoma may arise in adenomyosis or invade from a deep focus of adenomyosis. In this situation, the depth of invasion should be measured from the junction of the adenomyosis and myometrium to the deepest area of invasive carcinoma. (This figure was published in Uterine Pathology, Copyright Cambridge University Press, 2012).

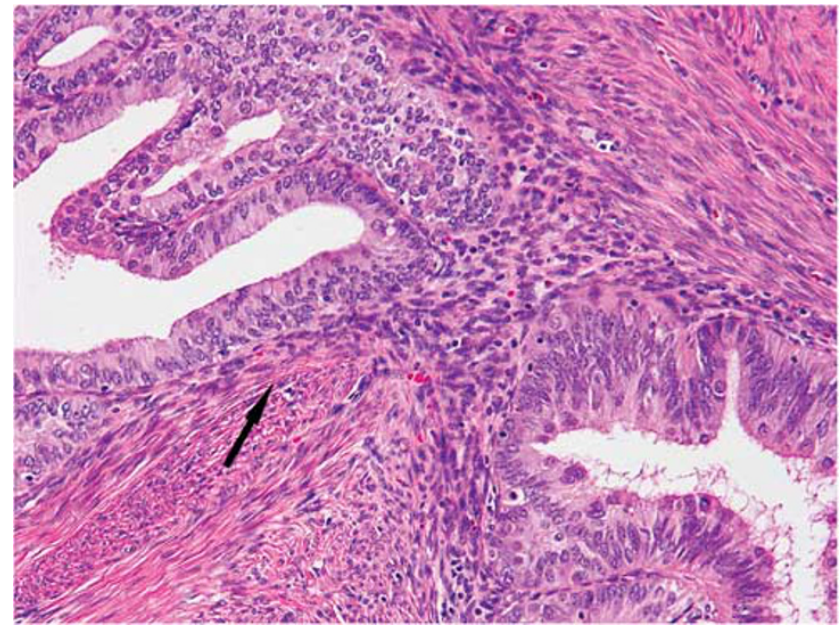

Figure 3 Metaplastic endometrial stroma. Note the transition between typical-appearing endometrial stroma and fibrillary, myoid stroma (indicated by the arrow). (This figure was published in Uterine Pathology, Copyright Cambridge University Press, 2012).

attenuation may mimic the appearance of endothelial cells, such that distinction between myometrial invasion and lymphovascular invasion becomes difficult. Furthermore, when there is true

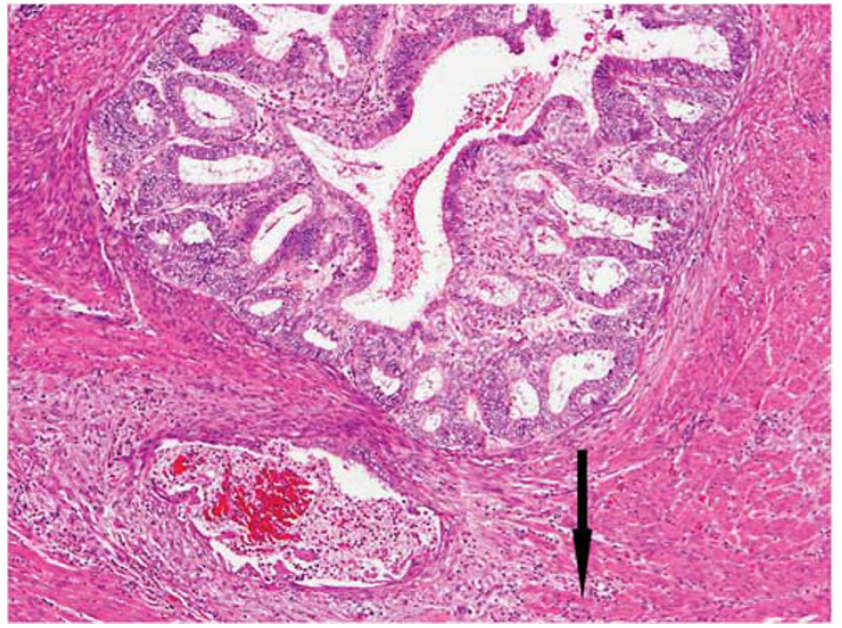

Figure 4 Focal myometrial invasion adjacent to adenomyosis. The arrow indicates my proposal for measuring depth of myometrial invasion.

lymphovascular invasion, a frequent finding in MELF invasion, the intravascular tumor cells may retain a histiocytoid appearance, unlike most of the overlying tumor (Figure 8). MELF invasion is 


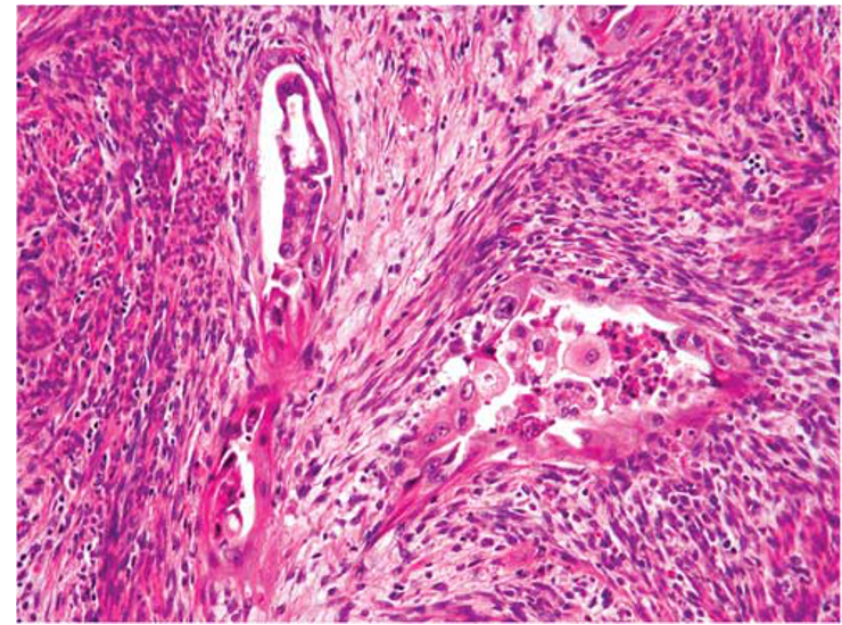

Figure 5 Myoinvasive endometrioid adenocarcinoma with a microcystic, elongated, and fragmented pattern (MELF). Note the histiocyte-like tumor cells, attenuated and squamous metaplastic tumor cells, periglandular stromal pallor, and aggregates of neutrophils. (This figure was published in Uterine Pathology, Copyright Cambridge University Press, 2012).

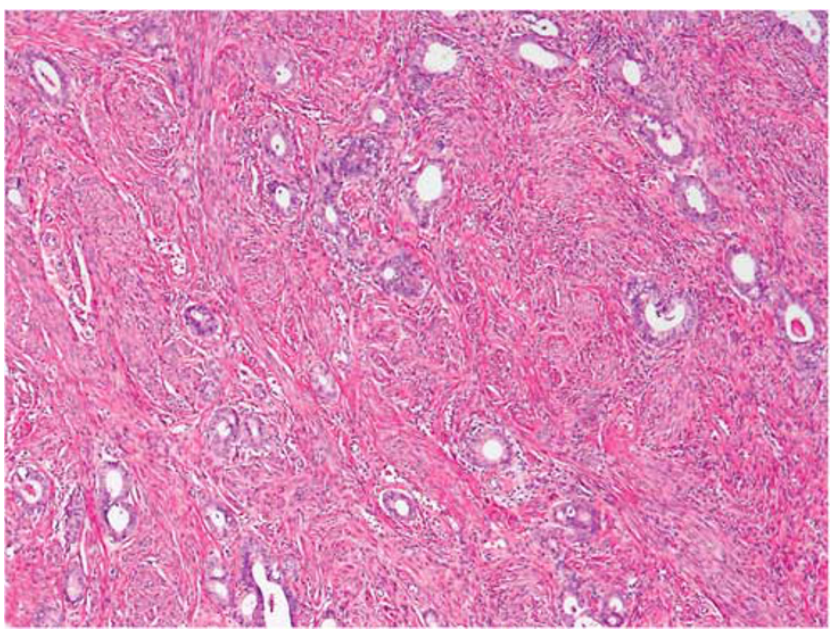

Figure 6 Myoinvasive endometrioid adenocarcinoma with an 'adenoma malignum-like' pattern. Well-differentiated endometrioid glands are dispersed throughout the myometrium and are not associated with a desmoplastic host response. (This figure was published in Uterine Pathology, Copyright Cambridge University Press, 2012).

statistically associated with lymphovascular invasion and metastasis to regional lymph nodes, but it has not yet been shown to be an independent prognostic indicator. MELF has been shown to be a consequence of epithelial-to-mesenchymal transition present at the invasive front of endometrial carcinomas, with the loss of epithelial cell adhesion molecules such as E-cadherin. ${ }^{11,12,16}$ It is possible that this pattern is over-represented in tumors with defective MLH1 expression. ${ }^{18}$

The adenoma malignum-like invasion pattern displays variable numbers of well-formed endometrioid

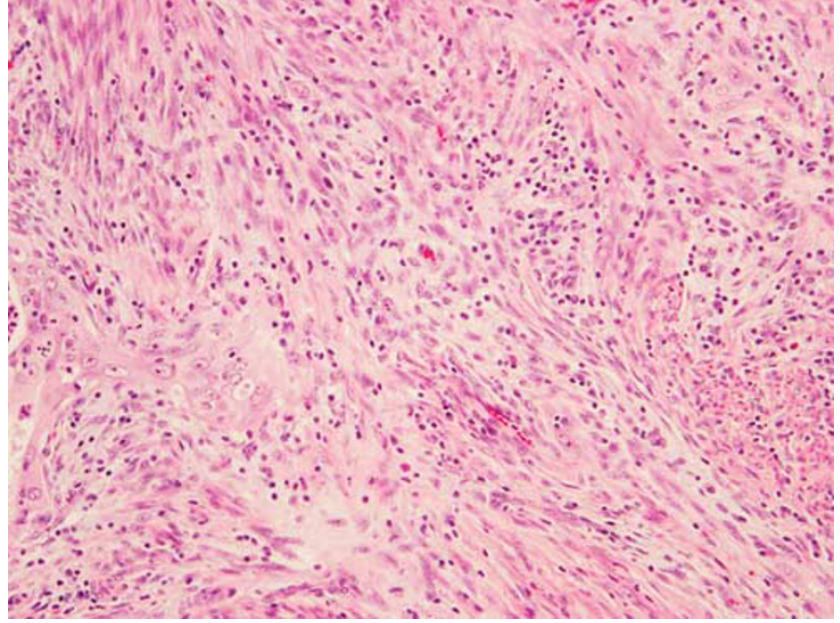

Figure 7 Microcystic, elongated, and fragmented (MELF) pattern invasion with a prominent myxoinflammatory stromal reaction. The myxoinflammatory reaction may distract from the presence of metaplastic tumor cells.

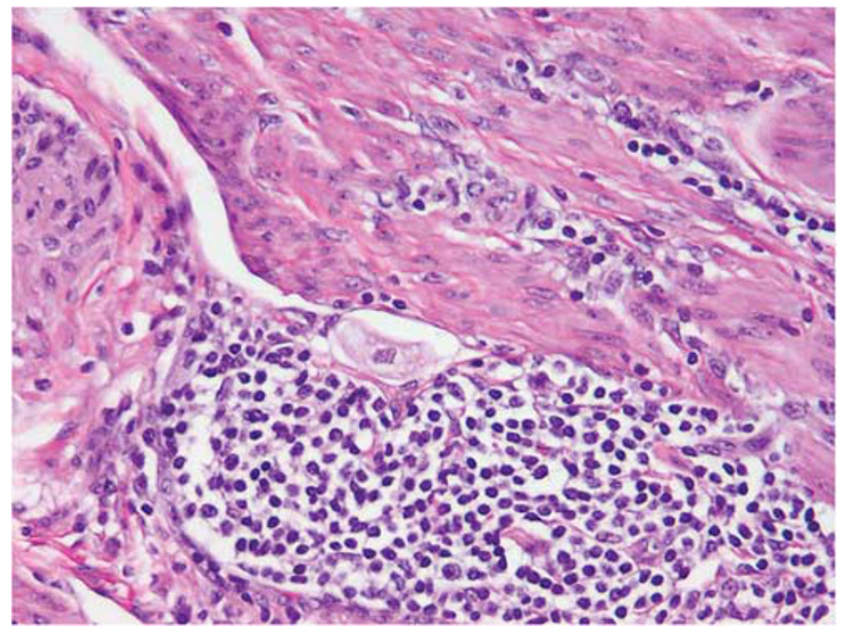

Figure 8 Lymphovascular invasion by a histiocyte-like tumor cell. (This figure was published in Uterine Pathology, Copyright Cambridge University Press, 2012).

glands haphazardly distributed throughout the myometrium, but a stromal response to invasion is either minimal or absent. ${ }^{14,19}$ This can be distinguished from adenomyosis by virtue of the chaotic arrangement of neoplastic glands, rather than the focal or multifocal clustered neoplastic glands surrounded by endometrial stroma that is characteristic of adenomyosis. The adenoma malignum-like pattern of invasion probably does not have prognostic significance when compared with tumors with conventional forms of myometrial invasion. It should also be noted that despite this entity's name, it bears no clinical or pathological relationship to minimal deviation adenocarcinoma of the endocervix. The only feature that 
adenoma malignum-like invasion shares with minimal deviation adenocarcinoma of the endocervix is the presence of invasive well-differentiated glands without an accompanying stromal response.

\section{Lymphovascular Invasion}

The recognition of lymphovascular invasion is usually straightforward. Both the presence of lymphovascular invasion and its extent are prognostically important. Foci of lymphovascular invasion should be sought at the advancing edge of the tumor as it invades myometrium. Occasionally, with serous carcinomas, one can confidently diagnose lymphovascular invasion within an endometrial polyp or within endometrial stroma. Otherwise, foci suspicious for lymphovascular invasion should not be interpreted as being 'positive for lymphovascular invasion' when intravascular tumor is found within the tumor itself. Distinction of the pseudoendothelial appearance of MELF from true lymphovascular invasion can be accomplished with one of the endothelial immunohistochemical markers, such as FLI-1, podoplanin, and/or CD31.

One of the most challenging differential diagnoses involves distinguishing lymphovascular invasion from artifactual tissue displacement (Figure 9). It has been reported that artifactual tissue displacement into myometrial vessels, spaces not lined by endothelium, fallopian tube lumen, and peritoneal washings is seen more commonly in laparoscopic and robotic operative procedures compared with open, or traditional, operative approaches. ${ }^{20-24}$ Although the phenomenon was originally considered solely a result of uterine manipulation and tumor fragmentation, it has also been reported that both surgeons and pathologists are responsible for this artifactual phenomenon. It is currently thought that uterine manipulation and tumor fragmentation resulting from the operative procedure presents the pathologist with friable tumor that can be dragged through the tissue during prosection. Fixation for several hours in formalin before prosection has been shown to minimize the occurrence of these artifacts. ${ }^{21}$ Clues pointing to the presence of artifact include finding neoplastic and non-neoplastic endometrium, oftentimes crushed and distorted, as well as stroma in vessels and non-endothelial-lined spaces of varying sizes close to the tumor and in distant sections. Occasionally, it may be impossible to confidently diagnose lymphovascular invasion in the presence of such artifact.

\section{Cervical Involvement}

Impediments to an accurate diagnosis of cervical stromal invasion are largely due to the absence of anatomical boundaries, or definitions thereof that rigidly separate cervix from lower uterine segment and endocervical mucosa from cervical stroma

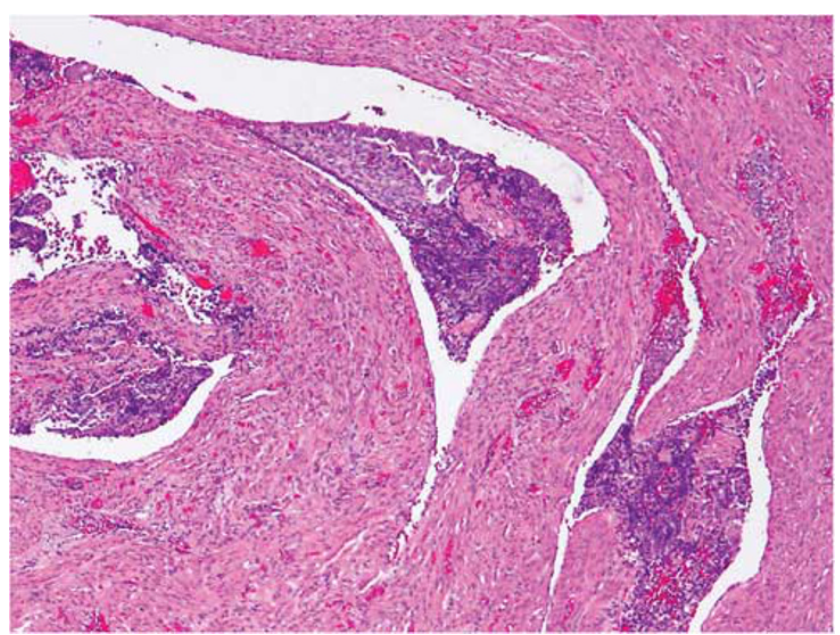

Figure 9 Artifactual displacement of tumor and non-neoplastic elements. (This figure was published in Uterine Pathology, Copyright Cambridge University Press, 2012).

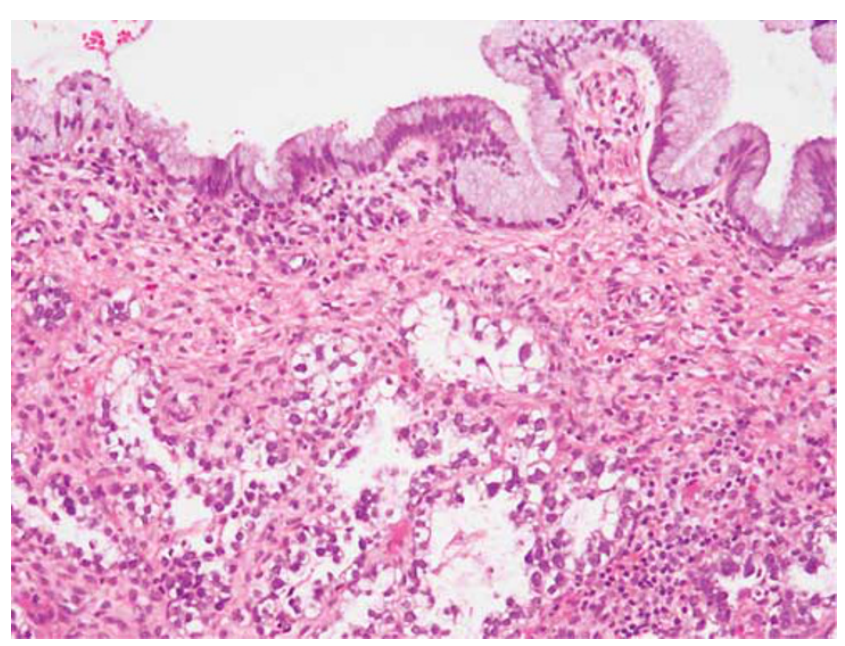

Figure 10 Cervical stromal invasion. This example shows clear cell carcinoma of endometrium invading cervical stroma. (This figure was published in Uterine Pathology, Copyright Cambridge University Press, 2012).

(Figures 10 and 11). These are some of the types of distinctions responsible for disappointing rates of interobserver agreement. ${ }^{25,26}$ One can be confident that cervical involvement is present when the neoplastic focus is bounded on two sides by normal endocervical glands (proximally and distally), and that stromal invasion is present when the invasive focus underlies normal ectocervix or endocervical glands. Tumors placed at the junction of the endocervix and lower uterine segment cannot be said to involve cervix with certainty when the tumor involves lower uterine segment and is bounded by endocervical tissue only distally. It can also be difficult to distinguish extensive endocervical mucosal involvement from cervical stromal invasion. An 

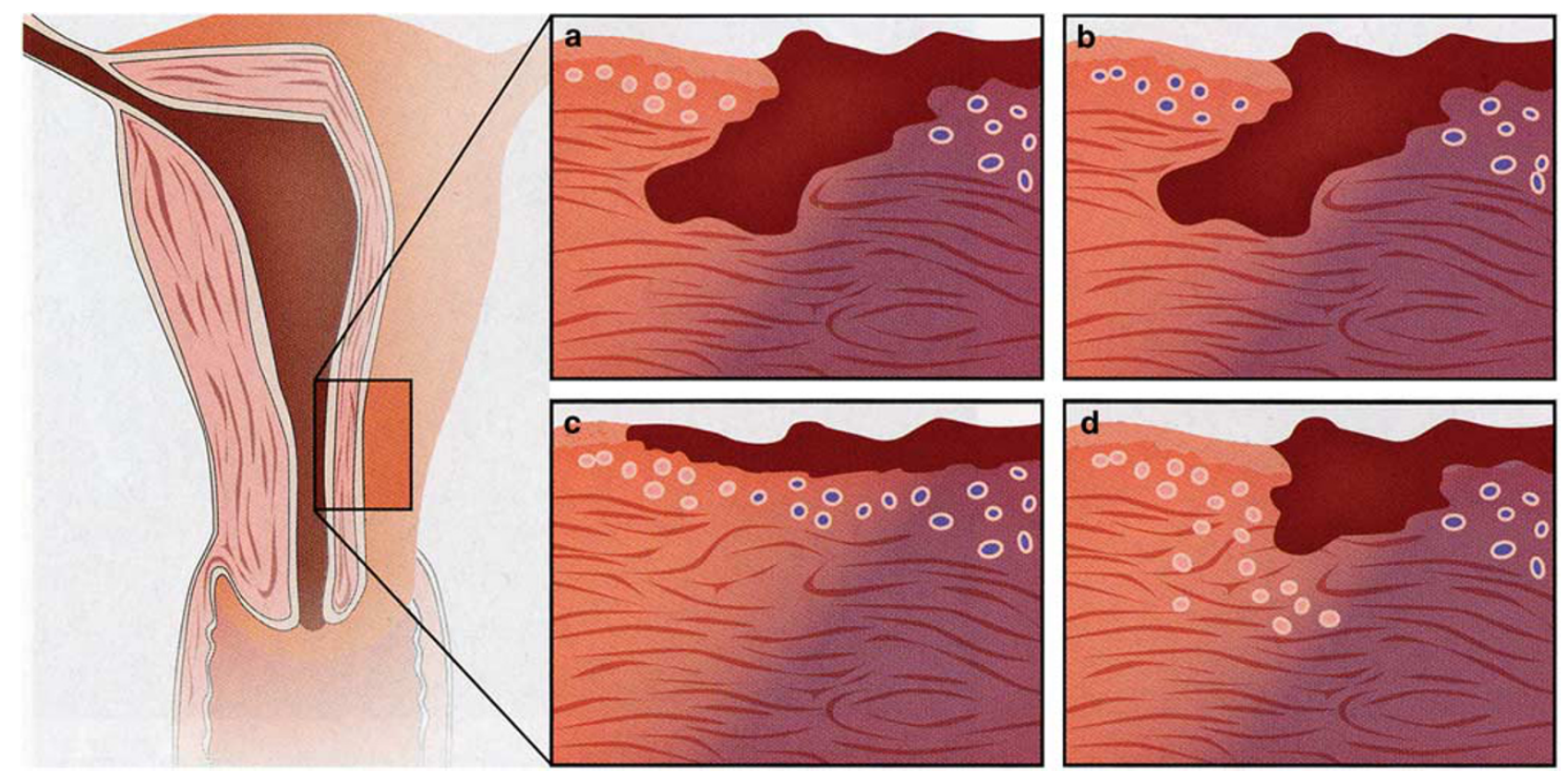

Figure 11 Assessment of cervical involvement by endometrial carcinoma. Endometrial glands, unlike endocervical glands, are depicted with blue centers. (a) Cervical stromal invasion must clearly involve endocervical stroma and not lower uterine segment. This is depicted by the presence of carcinoma within cervical stroma beneath endocervical glands. (b) In this panel, endometrial carcinoma invades the lower uterine segment, but not the cervix. Note endometrial glands bordering either side of the invasive front. Because the myometrium in the lower uterine segment is often more fibrous in appearance than in the fundus, the distinction between cervical stroma and lower uterine segment can be difficult without glandular landmarks. (c) In this panel, the endometrial cancer involves the cervix, but does not extend beyond the superficial normal endocervical glands and so does not represent stromal invasion. I would not consider this mucosal involvement, which no longer features in the current FIGO staging scheme. (d) In this panel, the adenocarcinoma extends beyond the level of the superficial cervical glands, but not beyond the deeper endocervical glands. Whether this pattern constitutes cervical stromal invasion is subject to interobserver disagreement. In the presence of substantial tumor involvement of cervical stroma at the depth depicted in this panel, I would not classify this stromal invasion. (This figure was published in Uterine Pathology, Copyright Cambridge University Press, 2012).

example of such a problematic case is a low-grade endometrioid adenocarcinoma that is exophytic or obscures normal endocervical mucosal landmarks but does not invade underlying stroma, and in which no stromal response to invasion is present (summarized in Figure 12). Thus, either irregular infiltration into stroma deep to the tumor or a stromal response at the pushing edge of the lesion would be required to diagnose cervical stromal invasion (Figure 13). In addition to these guidelines, it remains important to accurately distinguish embryological remnants and endocervical hyperplasias from endometrioid adenocarcinomas that involve cervical stroma with an adenoma malignum-like invasion pattern, ${ }^{27}$ as described earlier in this review. I believe in applying a high threshold for diagnosing cervical stromal invasion, as there are some recent data that suggest cervical stromal invasion alone is not independently associated with clinical outcome. ${ }^{28}$ Rather, it is possible that cervical stromal invasion co-exists with other factors known to influence poor outcomes, such as deep myometrial invasion, high tumor grade, and the presence of lymphovascular invasion.

Of note, rare endometrioid adenocarcinomas preferentially invade cervical stroma over myometrium, such that the location of the invasion does not inform the tumor type or primary site. ${ }^{27}$ Similarly, rare

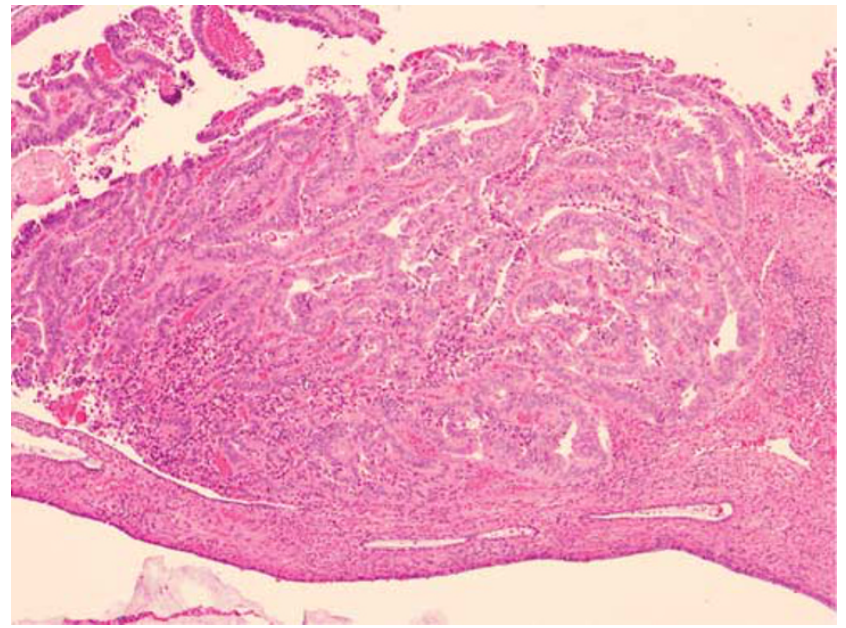

Figure 12 Endocervical mucosal colonization by low-grade endometrioid adenocarcinoma. Even though tumor replaces the mucosal compartment, there is neither invasion deep to nonneoplastic glands nor a stromal response to invasion at the tumor's base.

endocervical adenocarcinomas colonize lower uterine segment and the uterine corpus epithelium ${ }^{29}$ and may preferentially invade stroma and myometrium in those sites. Distinction between an endocervical 
adenocarcinoma preferentially involving corpus and an endometrioid adenocarcinoma preferentially involving cervix may require the use of immunohistochemistry.

\section{Synchronous Endometrial and Ovarian Carcinoma}

The coexistence of adenocarcinoma in endometrium and ovary presents difficulties for stage assignment, prognostication, and therapy. Synchronous adenocarcinomas of ovary and endometrium should be staged separately (usually FIGO stage I in each site), whereas metastasis from endometrium to ovary should be designated endometrial carcinoma, FIGO stage III. Guidelines were initially developed with the idea that synchronous tumors should behave as two low-stage tumors rather than one high-stage tumor. In other words, the guidelines segregate tumors into one clinically indolent category (i.e., 'synchronous') and another potentially aggressive category (i.e., 'metastatic'). Specifically, guidelines were not developed by determining clonal relationships between tumors in endometrium and ovary; in fact, several investigators have reported clonal relationships between 'synchronous' endometrial and ovarian carcinomas. ${ }^{30-32}$ Although

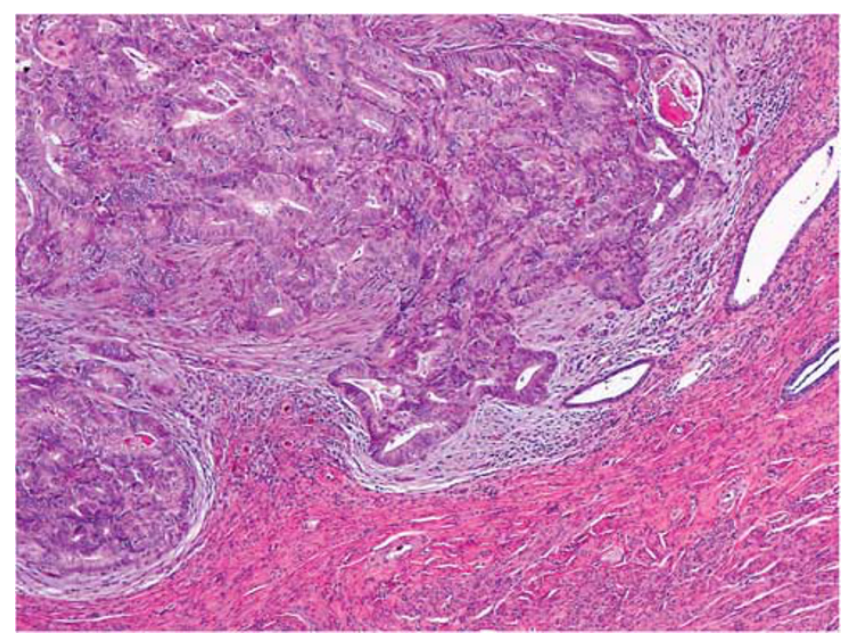

Figure 13 Endocervical mucosal and stromal invasion. Cervical stromal invasion is confirmed by the presence of a stromal response at this tumor's irregularly shaped leading edge. such tumors may represent metastatic tumors from a genotypic perspective, there is as yet no clinical evidence that shared clonality in this context has clinical value.

There are two characteristic types of synchronous endometrial and ovarian carcinomas. The most common occurs in young patients, with paradoxically histologically identical tumors in both sites, and a large, unilateral ovarian tumor. ${ }^{33-38}$ Table 2 summarizes the guidelines for recognizing synchronous endometrial and ovarian carcinoma when histology is similar in both organs. Most of these tumors are sporadic, although a small percentage is attributable to Lynch syndrome. ${ }^{39}$ The less common type of synchronous carcinoma involves tumors of different types or, perhaps, grades. A good number of these tumors are attributable to Lynch syndrome, particularly when either pure or mixed clear cell carcinoma is present in either site. ${ }^{40,41}$

For endometrial endometrioid carcinomas, typical patterns of metastasis to ovaries include bilateral involvement measuring $<10 \mathrm{~cm}$, ovarian surface involvement, and a nodular growth pattern that preserves ovarian stroma between nodules (Table 3). For serous carcinomas that involve endometrium and ovary, most pathologists default to a diagnosis of metastasis from endometrium to ovary, especially when the volume of tumor in endometrium exceeds that in ovary, but this paradigm will probably change as we gain a better understanding of the specificity of WT1 immunostaining and intraepithelial serous carcinoma in endometrium and/or fallopian tube. ${ }^{42-54}$ Along these lines, there is now increasing interest in being able to recognize 'drop metastasis' of serous carcinoma from uterine adnexa to uterine corpus or cervix (Figure 14). There is little evidence supporting the idea that synchronous serous

Table 3 Guidelines for recognizing metastatic endometrioid carcinoma of endometrium to ovary

Small ovaries $(<10 \mathrm{~cm})$

Multinodular configuration

Bilateral ovarian involvement

Surface involvement

Destructive stromal invasion

No associated endometriosis or müllerian borderline tumor

Table 2 Guidelines for recognizing synchronous endometrial and ovarian carcinoma with similar histology

Endometrium

Low-grade endometrioid, no or superficial myometrial invasion and no lymphovascular invasion

Ovary

Low-grade endometrioid

Large, unilateral tumor

Expansile invasion

Associated endometriosis, müllerian borderline tumor

Patient $<50$ years

Abbreviation: FIGO, International Federation of Gynecology and Obstetrics. 

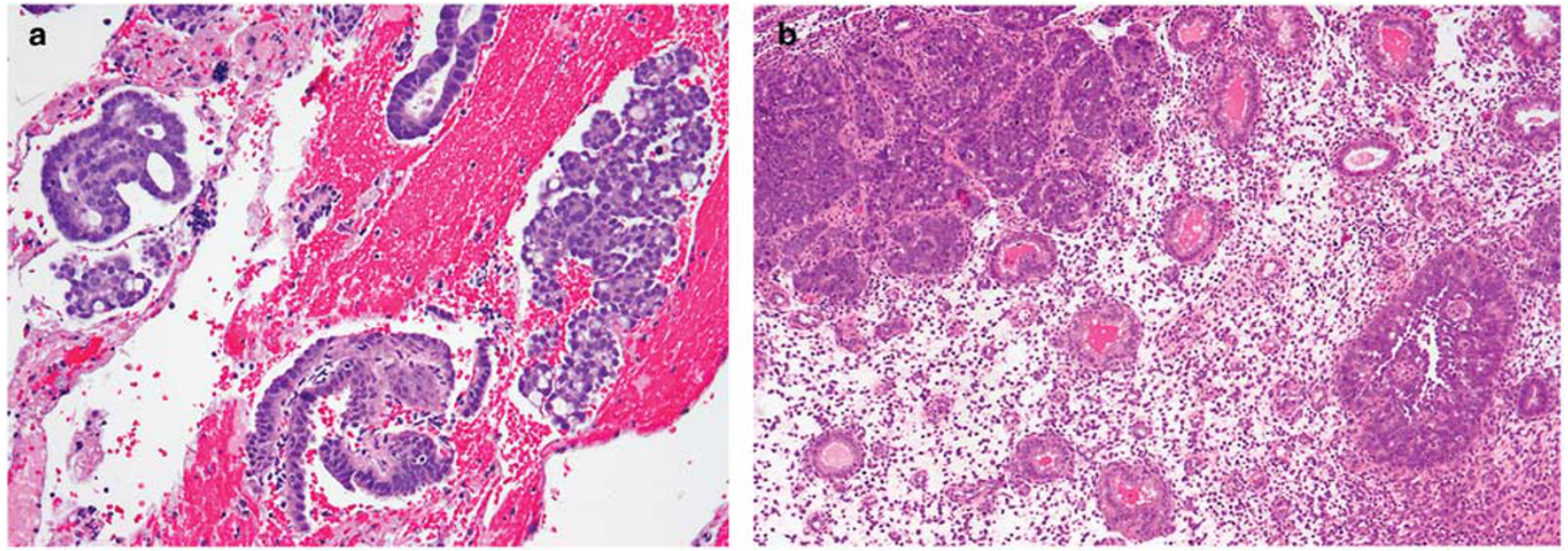

Figure 14 Drop metastases. (a) Small, dissociated fragments of serous carcinoma amidst atrophic endometrium; (b) A drop metastasis from a high-grade serous carcinoma of fallopian tube that involves secretory endometrium. (This figure was published in Uterine Pathology, Copyright Cambridge University Press, 2012).

Table 4 Serous carcinomas involving endometrium and ovary: Guidelines for recognizing metastatic serous carcinoma from endometrium to ovary

Patient older than 60 years

Endometrial polyp with serous carcinoma

No or minimal fallopian tube involvement

More tumor in endometrium than ovary

Negative WT1

Table 5 Guidelines for recognizing drop metastasis from adnexal serous carcinoma to endometrium

Extensive fallopian tube involvement, including serous tubal intraepithelial carcinoma

Small fragments of nested tumor in curettage admixed with non-neoplastic endometrium

Multicentric endometrial deposits

Positive WT1

carcinomas of endometrium and ovary exist in any significant numbers. Table 4 summarizes guidelines for diagnosing endometrial serous carcinoma metastatic to ovary(ies) and Table 5 summarizes guidelines to recognize drop metastases (metastasis from ovarian or fallopian tube serous carcinoma to endometrium or cervix).

Note that Tables 2,3,4 and 5 present guidelines rather than diagnostic criteria. In many cases, it is impossible to distinguish synchronous from metastatic patterns with certainty. Given the following example, it is extremely difficult to assign the tumor to either category: a deeply invasive FIGO grade 1 endometrioid adenocarcinoma of endometrium with a large, unilateral ovarian tumor involving ovarian surface in an older woman. A good practice is to list the features supporting synchronous or metastatic disease and inform the clinician that a more precise diagnosis cannot be made based on the evidence at hand.

\section{Lymph Node Metastasis}

It is usually easy to determine when endometrial carcinoma is present in lymph nodes, but interest in MELF invasion, the appearance of associated histiocyte-like tumor cells in lymphatics and subcapsular sinuses, ${ }^{55}$ and the increasing use of cytokeratin immunohistochemistry for ultrastaging sentinel lymph nodes have made this issue more challenging (Figure 15). Ultra-staging with cytokeratin immunohistochemistry has revealed a small, but troublesome, number of cases with low-volume metastases, including single keratin-positive cells, isolated tumor cells, and micrometastases, using definitions from breast pathology. ${ }^{56-61}$ Although many pathologists and gynecologists consider these scenarios to represent FIGO stage IIIC endometrial carcinoma, some caution is warranted. Interestingly, isolated tumor cells tend to be associated with clinically stage I, FIGO grade 1 endometrioid adenocarcinoma with or without MELF invasion, which in the absence of isolated tumor cells would be considered low- or, at most, intermediate-risk disease. This contrasts with the far more common situation in which FIGO stage IIIC disease is seen in conjunction with high-grade tumor, nonendometrioid histology and, frequently, extensive myometrial invasion. Aside from the uncertain clinical significance of isolated tumor cells in otherwise low-risk disease, considering these patients to have FIGO stage IIIC carcinoma may contaminate a category about which much is known and where the delivery of adjuvant therapy 

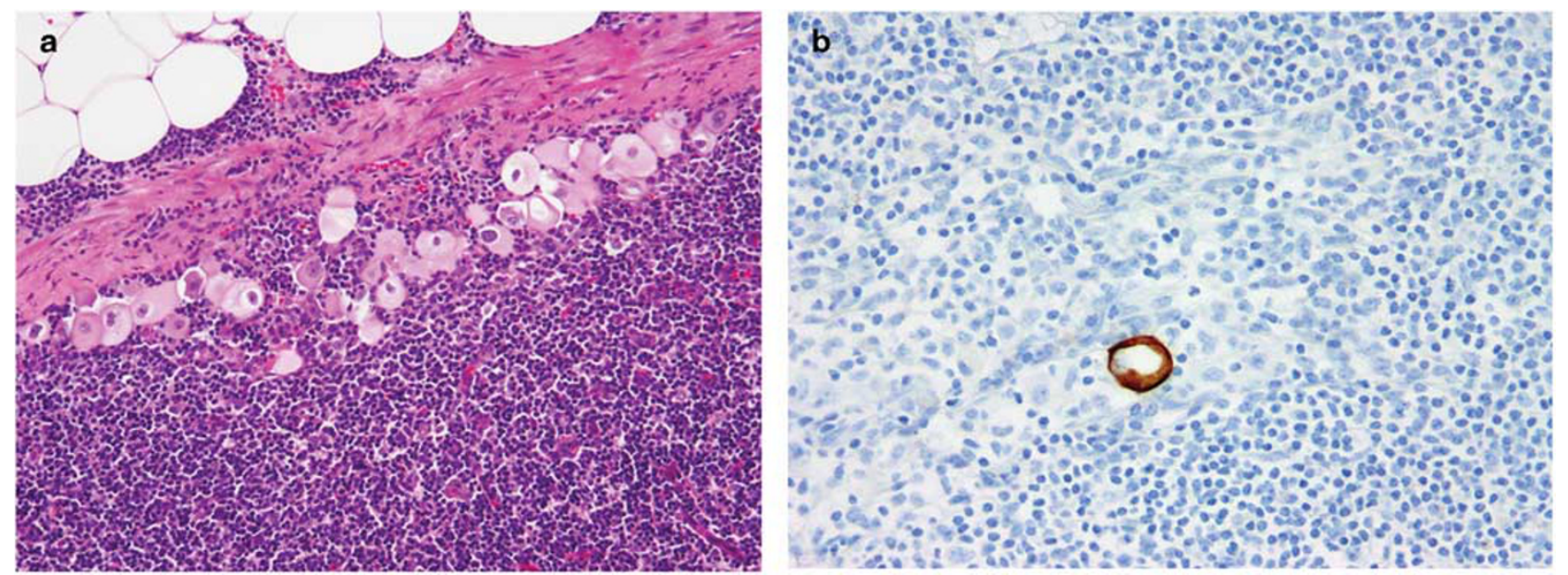

Figure 15 Lymph node metastasis by histiocytoid tumor cells. (a) Metastatic endometrioid adenocarcinoma with a histiocytoid appearance; (b) A single, cytokeratin-positive cell in a sentinel lymph node. (This figure was published in Uterine Pathology, Copyright Cambridge University Press, 2012).

constitutes the standard of care. It is therefore considered controversial by some pathologists whether to use ultrastaging to identify low-volume metastases.

\section{Frozen section}

Frozen section to determine tumor grade and depth of myometrial invasion is used to guide the sampling of lymph nodes for therapy and prognostication. This is a very familiar concept for US pathologists, although it is used less frequently in other parts of the world. Frozen section performed for this purpose is time consuming, may be inaccurate in as many as $15 \%$ of cases, ${ }^{7-9}$ introduces artifacts by serial sectioning before formalin fixation, is justified by data that have been accrued in the postoperative setting (rather than pre- or intraoperatively), and is applied in an empirical fashion by gynecologists. A hysterectomy (i.e., postoperative) diagnosis of myometrial-invasive FIGO grade 2 or grade 3 carcinoma places the patient into an intermediate-to-high clinical risk category (i.e., the patient may have lymph node metastases), as does $>50 \%$ invasion when the tumor is FIGO grade 1. ${ }^{62,63}$ Tumor size assessed intraoperatively is also used in one popular algorithm. ${ }^{64}$ This information has been used to guide the performance of lymph node dissection after intraoperative examination.

The decision to request frozen section and how to use the data also vary by surgeon. This is due to a lack of consensus in the gynecology community regarding whether lymph node dissection is therapeutic, whether lymph node sampling can replace lymph node dissection, and what constitutes an adequate lymph node dissection. Many gynecologists also work under the erroneous impression that the distinction between FIGO grades 1 and 2 is reproducible and has the same significance in the pre- and intra-operative setting as it does in the postoperative setting; they also tend to believe that the assessment of the depth of myometrial invasion is reproducible. Review of the pertinent literature indicates that lymph node dissection is not therapeutic, ${ }^{65,66}$ but it is preferable to lymph node sampling, ${ }^{67,68}$ as a more accurate tumor stage can be ascertained. In some studies, adequate lymph node staging is set at approximately 10-15 lymph nodes, ${ }^{69,70}$ although the requirement to dissect paraaortic lymph nodes in addition to pelvic lymph nodes in every case is still controversial. Without knowing a patient's lymph node status, there is indeed a relationship between tumor grade and depth of invasion and, secondarily, with lymph node metastasis, but once a formal surgical stage is assigned, the clinical meaning of separating FIGO grades 1 and 2 loses value. ${ }^{71}$ Intraoperative macroscopic evaluation of the depth of myometrial invasion is even less accurate than frozen section ${ }^{72-77}$ and similarly introduces artifact.

Most frozen sections to determine depth of invasion are performed in patients with a known diagnosis of atypical hyperplasia/endometrial intraepithelial neoplasia (EIN) or FIGO grade 1 endometrioid adenocarcinoma, because these neoplasms are the most common and the extent of myometrial invasion, if present, informs the decision to perform lymph node sampling or dissection. Despite the fact that between 5 and $15 \%$ of these neoplasms show substantially invasive carcinoma at frozen section, only $2 \%$ metastasize to lymph nodes. ${ }^{62,78,79}$ Given a hypothetical group of 100 patients with endometrial cancer, one would have to perform 100 frozen sections (including preparation of as many as 200 frozen tissue blocks or more) and up to 15 lymph node dissections to find the 2 patients who might be more accurately staged by having had a lymph node dissection performed. Whether these 2 patients 
necessarily benefit from adjuvant therapy is unknown, but some gynecologists estimate that the use of adjuvant therapy in this setting might prevent $50 \%$ of recurrences, thereby possibly benefitting 1 of 100 theoretical patients. These findings have led to the development of alternatives to frozen sections.

Two alternatives obviate the need for frozen section: performance of lymph node dissection in every patient, without regard to pre-operatively assessed tumor type or grade, and the use of sentinel lymph node mapping. Performing lymph node dissection for every patient with endometrial cancer has met considerable resistance because of the risk of lower extremity lymphedema, ${ }^{80}$ cost, increased operative time, need for a gynecologic oncologist instead of a generalist, and the number of blocks and slides generated. The sentinel lymph node mapping procedure therefore appears most attractive. Patients undergoing this procedure ${ }^{56-61}$ are accurately staged (at least in $\sim 90 \%$ ) without lymphadenectomy and are spared the risk of lymphedema. Before the procedure, blue dye and/or a tracer is injected into the lower uterine segment. Mapping at the time of surgery reveals the sentinel node(s). These lymph nodes are removed along with any enlarged lymph nodes and all blue dye/tracer-negative lymph nodes that are contralateral to sentinel lymph nodes. ${ }^{59}$ Frozen section is not performed because there is no intraoperative question to answer. The sentinel lymph nodes are breadloafed at 2-mm intervals and entirely submitted for microscopy. At Memorial Sloan Kettering Cancer Center, two hematoxylin and eosin (H\&E) slides are prepared, separated by 50 microns. Unstained slides corresponding to each $\mathrm{H} \& \mathrm{E}$ level are prepared, and one pan-cytokeratin immunohistochemical stain is performed at each level (i.e., two immunostained slides separated by 50 microns). Unlike the previous paradigm in breast cancer, patients are not brought back to the operating room for completion of the dissection when sentinel lymph nodes are positive for carcinoma. Problems with implementation, however, involve challenges with accurate mapping in patients with high body mass indexes (BMIs) and when the surgeon has insufficient experience with the mapping procedure. From the pathology perspective, one confronts problems when undue reliance is placed upon the use of cytokeratin immunohistochemical stains to detect low-volume metastasis, particularly because it has not been shown that survivals are comparable to macrometastasis (i.e., $>2 \mathrm{~mm}$ ) that can be visualized without immunohistochemistry. Other considerations are the theoretical possibility of mistaking benign mesothelial cells and Mullerian inclusions for low-volume metastatic carcinoma, and many pathology practices are reluctant to perform more immunohistochemical stains, as reimbursements for this procedure are limited. Therefore, it may be reasonable to forego the use of cytokeratin immunohistochemistry for ultrastaging, provided that multiple recut sections are examined carefully.

\section{Lynch syndrome}

In women with Lynch syndrome, the risk of developing endometrial and ovarian cancer exceeds that of colorectal cancer; and in patients who develop both gynecological and intestinal carcinomas, a small majority develops gynecological cancer first. ${ }^{81}$ Identifying which endometrial cancer patients have Lynch syndrome saves lives by instituting surveillance colonoscopies in affected patients and relatives; it has been reported that surveillance for colorectal carcinoma in known Lynch syndrome patients reduces mortality from colorectal carcinoma by $65 \%{ }^{82-84}$ Performing prophylactic hysterectomy and bilateral salpingo-oophorectomy in known carriers is also advantageous. Although Lynch syndrome is somewhat heterogeneous, there are some important general differences between Lynch syndrome-associated ${ }^{85-90}$ and sporadic endometrial cancer (Table 6). Patients with Lynch syndromeassociated endometrial carcinoma are younger and have lower BMIs. The prognostic and therapeutic implications of Lynch syndrome-associated endometrial carcinoma are, as of yet, uncertain. Between 2 and $5 \%$ of endometrial carcinomas are attributable to Lynch syndrome. Although the incidence of Lynch syndrome increases with age, the prevalence decreases significantly because of the strikingly increased incidence of sporadic endometrial carcinoma as patients age. Nevertheless, it has been reported that $\sim 10 \%$ of patients with endometrial carcinoma under 40 years of age have Lynch syndrome.

Lynch syndrome is currently diagnosed in the presence of one of the following: (1) germline mutation of one of the DNA-mismatch repair genes (MLH1, PMS2, MSH2, and MSH6); (2) germline EPCAM mutation; and (3) constitutive epimutation. The first scenario, germline mutation of one of the DNA-mismatch repair genes, is by far the most commonly encountered abnormality among Lynch syndrome patients. A carrier has one copy of a defective DNA-mismatch repair gene in somatic cells throughout her body. A second 'hit' occurs in the target organ, such as the endometrium, leading to loss of a functioning DNA-mismatch repair protein, thereby interfering with physiologic DNA-mismatch repair. DNA-mismatch repair is accomplished by two sets of heterodimers, MLH1 with PMS2 and MSH2 with MSH6. Loss of a functioning DNAmismatch repair protein almost always results in the

Table 6 Comparison of Lynch syndrome-associated and sporadic endometrial carcinoma

\begin{tabular}{lcc}
\hline Characteristic & Lynch syndrome associated & Sporadic \\
\hline Age at presentation & $>40$ years & $>60$ years \\
Body mass index & Low & High \\
Clinical behavior & Unknown & Baseline \\
\hline
\end{tabular}


Table 7 Comparison of Lynch syndrome-associated endometrial carcinoma and colorectal carcinoma

\begin{tabular}{lcc}
\hline Characteristic & Endometrial carcinoma & Colorectal carcinoma \\
\hline Age at presentation & $>40$ years & $>35$ years \\
DNA-mismatch repair mutations & hMSH $>h M S H 2>h M L H 1>$ PMS 2 & $h M L H 1=h M S H 2>>h M S H 6$ \\
Microsatellite instability rate & $30 \%$ & $>90 \%$ \\
Family history & $30 \%$ & $80 \%$ \\
\hline
\end{tabular}

loss of immunohistochemical expression of the correspondingly mutated DNA-mismatch repair protein, and usually also its partner (i.e., MLH1 along with PMS2; and MSH2 along with MSH6). Loss of a functioning DNA-mismatch repair protein is also usually followed by the acquisition of multiple microsatellites that do not match those present in tissues unaffected by cancer (microsatellite instability (MSI)). Germline EPCAM mutation leads to downregulation of MSH2 with similar downstream effect as when the MSH2 gene is mutated, while constitutive epimutation involves widespread methylation of genes throughout the body, including MLH1, the consequence of which is similar to sporadic MLH1 methylation or mutation. Currently, Lynch syndrome can therefore only be diagnosed by studying tissues unaffected by carcinoma, such as blood, because these would contain the germline mutations that define the entity without all of the secondary changes acquired by the tumor in the target site.

Regarding the heterogeneity of Lynch syndrome, it should be noted that the gene mutation present has important implications. Mutation type is associated with the relative risk of developing endometrial carcinoma and extra-gynecologic cancers, the age at which carcinoma develops, and easily detected MSI. ${ }^{85-90}$ Mutations in MSH6 are more prevalent in Lynch syndrome-associated endometrial carcinoma than in Lynch syndrome-associated colorectal carcinoma, and this accounts for some of the differences between them (Table 7). For example, MSH6 germline mutation carriers present at an older age and tend to develop endometrial carcinoma more often than colorectal carcinoma. This indicates that the approach to Lynch syndrome screening in endometrial cancer should differ from that in colorectal cancer. The average patient with Lynch syndromeassociated endometrial carcinoma is older than someone with Lynch syndrome-associated colorectal carcinoma, more likely to have an MSH6 mutation, less likely to have an MSI-high (MSI-H) tumor, and less likely to have a personal or family history of Lynch syndrome-associated carcinomas.

Although it is currently not feasible to perform germline sequencing to detect mutated DNAmismatch repair genes in every endometrial carcinoma patient, universal germline sequencing, requiring patient consent, may become the standard Lynch syndrome workup. In the interim, we will rely on the use of immunohistochemistry to detect
DNA-mismatch repair proteins (DNA MMR IHC). It has been shown that DNA MMR IHC is $\sim 90 \%$ sensitive and $90 \%$ specific for a DNA-mismatch repair abnormality; $;^{90-93}$ and as of 2009, DNA MMR IHC followed by targeted germline sequencing was the most efficient and cost-effective way to determine which endometrial cancer patients have Lynch syndrome. ${ }^{91}$ For example, if DNA MMR IHC shows loss of expression of MSH2 and MSH6, the germline sequencing will focus on those genes specifically, without having to sequence MLH1 and PMS2. Thus, DNA MMR IHC is an indirect way of screening for Lynch syndrome because confirmatory germline testing is still required for a Lynch syndrome diagnosis.

Why is DNA MMR IHC only an indirect direct test for Lynch syndrome? DNA MMR IHC detects almost any abnormality in DNA MMR gene expression, whether or not the insult is germline (i.e., due to Lynch syndrome). In fact, $20-30 \%$ of endometrial carcinomas are MSI-H and show abnormal DNA MMR IHC, yet only $2-5 \%$ of patients have Lynch syndrome, as mentioned previously. The most common reason for DNA MMR IHC abnormal/no germline DNA-mismatch repair mutation is methylation of the MLH1 promoter, found only in the target organ's cancer. Comparatively less is known about the occasional somatic DNA-mismatch repair mutation found only in the target organ's cancer. If a cancer cell harbors one allele with a somatic DNAmismatch repair mutation, a second hit needs to occur before damaging the DNA-mismatch repair system. In many cases, a second somatic DNAmismatch repair mutation occurs.

It is debatable whether or not to test all endometrial cancers with DNA MMR IHC or to restrict immunohistochemical testing to certain categories of patients and carcinomas (i.e., selective screening). There is a growing consensus that, if resources permit, all endometrial carcinomas should undergo DNA MMR IHC testing because most selective screening schemes fail to detect all Lynch syndrome-associated endometrial carcinomas. ${ }^{94,95}$ To limit resource expenditure, an age limit, perhaps 65 years, can be set, beyond which the ratio of Lynch syndrome-associated endometrial carcinoma to sporadic endometrial cancer falls precipitously and to use a two-marker DNA MMR IHC panel (i.e., MSH6 and PMS2) rather than the more traditional four-marker panel (MLH1, PMS2, MSH2, and MSH6). ${ }^{94}$ To limit resource expenditure further, 
more restrictive screening paradigms can be used, such as the one in use at Memorial Sloan Kettering Cancer Center ${ }^{40}$ (Table 8; Figures 16,17,18), which has been validated externally. ${ }^{96}$ This algorithm utilizes DNA MMR IHC testing in all endometrial cancers from patients younger than 50 years of age, when the personal or family history suggests Lynch syndrome and when a one of a variety of different tumor morphological and topographic features is present. The important morphological and topographic features that appear in this algorithm have been described in a series of Lynch syndromeassociated and MSI-H endometrial carcinomas. The general rule is as follows: as more restrictions are placed on screening and the lower the resource expenditure, the more Lynch syndrome patients will go unrecognized.

Loss of DNA-mismatch repair expression in tumor cell nuclei is equated with an abnormal DNA MMR IHC result. To score this pattern properly, there should be no tumor cell nuclear staining and an intact internal positive control, usually provided by non-neoplastic stromal cells, endothelium, and tumor-infiltrating lymphocytes (Figure 19). Table 9 displays the most common abnormal DNA MMR

Table 8 Algorithm for selective DNA-mismatch repair immunohistochemistry in use at Memorial Sloan Kettering Cancer Center

Age $<50$ years

Personal or family history suggestive of Lynch syndrome Dense peritumoral lymphocytes

Tumor infiltrating lymphocytes ( $>40$ /high power fields) Intratumoral heterogeneity

Multifocal endometrial tumors

Histological ambiguity

'Non-endometrioid' carcinoma in patients $<60$ years

Synchronous ovarian clear cell carcinoma

Lower uterine segment localization
IHC staining patterns, listed in order of decreasing frequency. Of note, the most common patterns involve loss of expression of two proteins together, either MLH1/PMS2 or MSH2/MSH6. Less frequently, one finds abnormal PMS2 with intact MLH1 or abnormal MSH6 without PMS2, all of which supports the use of a two-marker DNA MMR IHC panel consisting of PMS2 and MSH6 alone. Table 10 lists some common pitfalls in interpreting DNA MMR IHC. The two most common are the following: distinguishing weak or equivocal staining from abnormal staining (Figure 20) and misinterpreting DNA-mismatch repair-intact tumor-infiltrating lymphocytes as tumor cells with intact DNA-mismatch repair staining (Figure 21). In the first instance,

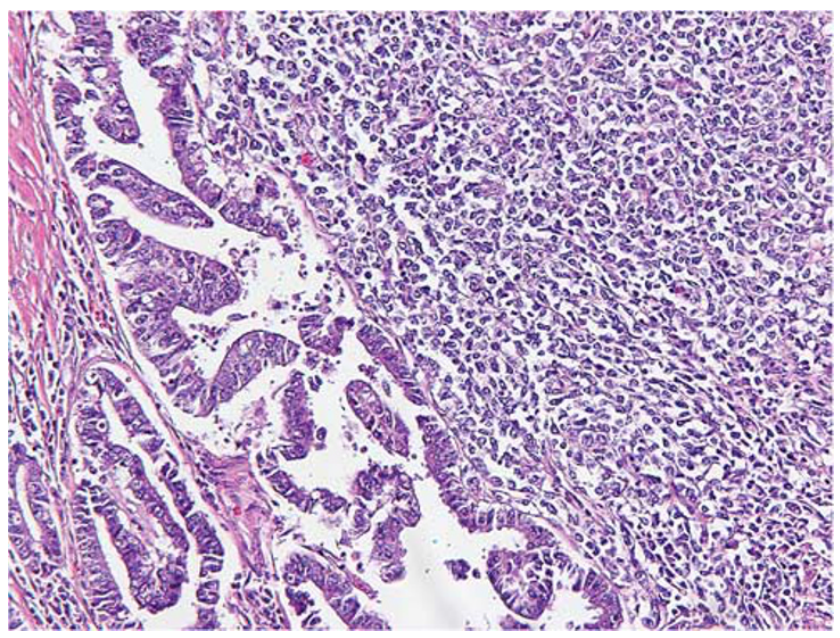

Figure 17 Intratumoral heterogeneity. This example is a dedifferentiated endometrial carcinoma. (This figure was published in Current Concepts in Gynecologic Pathology: Epithelial Tumors of the Gynecologic Tract, An Issue of Surgical Pathology Clinics, 1e, Copyright Elsevier, 2011).
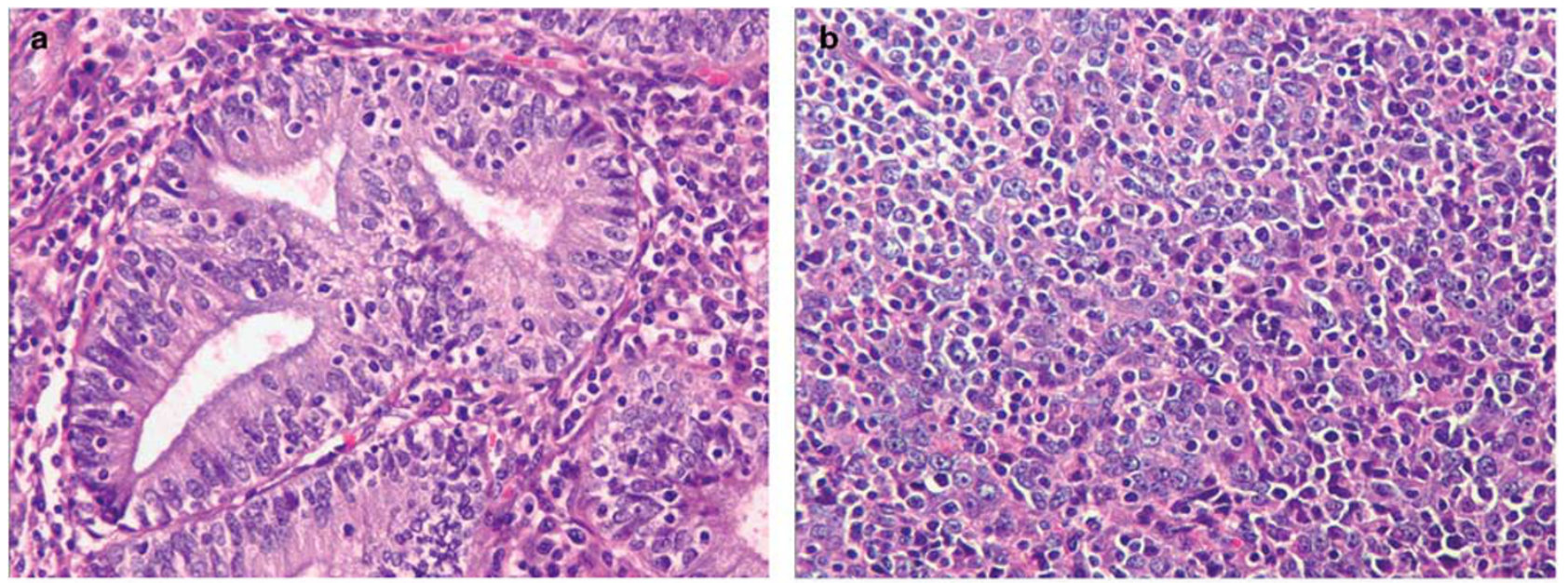

Figure 16 Tumor infiltrating lymphocytes (a and b). (This figure was published in Current Concepts in Gynecologic Pathology: Epithelial Tumors of the Gynecologic Tract, An Issue of Surgical Pathology Clinics, 1e, Copyright Elsevier, 2011). 
one should repeat the stain, show the stain to a knowledgeable colleague, study the immunohistochemistry for the partner protein (i.e., look at PMS2 if the MLH1 is equivocal), and/or choose another tumor block for staining. If the results remain equivocal, it is better to report the result as inconclusive rather than choosing either 'normal' or 'abnormal.' Distinguishing tumor-infiltrating lymphocytes from tumor cells is generally straightforward once one is aware of this type of pitfall. If one is unsure, leukocyte common antigen immunohistochemistry should be considered.

When the DNA MMR IHC shows abnormal MLH1 and PMS2 expression, the most commonly encoun-

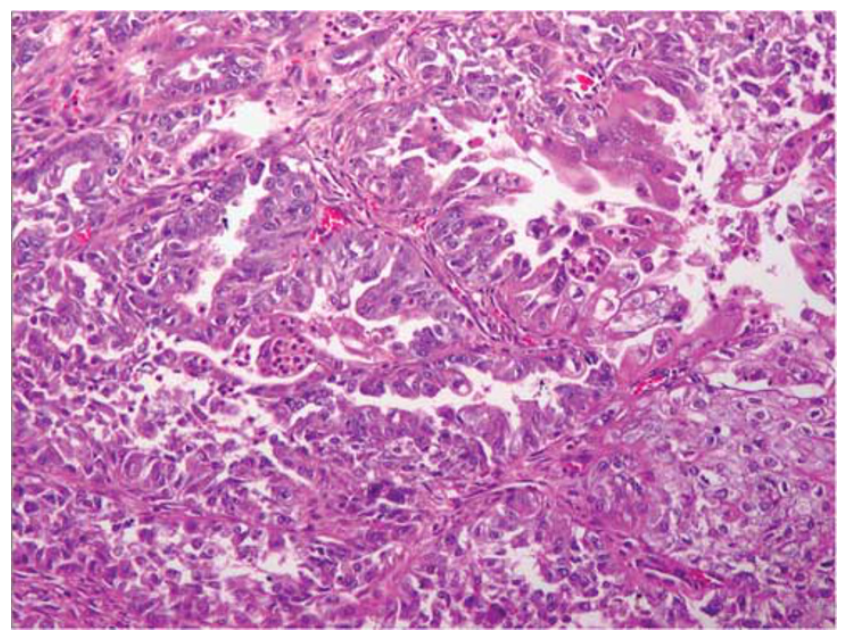

Figure 18 Morphologic ambiguity. The carcinoma is best categorized as endometrioid, but some features are reminiscent of serous or clear cell carcinoma. (This figure was published in Current Concepts in Gynecologic Pathology: Epithelial Tumors of the Gynecologic Tract, An Issue of Surgical Pathology Clinics, 1e, Copyright Elsevier, 2011). tered abnormal staining pattern, germline genetic testing should not be undertaken without first performing an MLH1 promoter methylation assay, as seen in Figure 22. Since the presence of MLH1 promoter methylation in the tumor signifies that the patient is at lowest risk of having Lynch syndrome, germline genetic testing is generally not performed. This underscores another important difference between MSI-H endometrial and colorectal carcinomas that are not Lynch syndrome associated. Most MSI-H colorectal carcinomas that are not associated with Lynch syndrome also have BRAF mutations that can be ascertained with immunohistochemistry or somatic mutation (tumor) testing. The MLH1 promoter methylation assay takes the place of BRAF testing in MSI-H endometrial carcinoma because $B R A F$ mutations are only rarely found in endometrial cancers.

Table 9 Commonly encountered DNA-mismatch repair immunohistochemical staining patterns ${ }^{\mathrm{a}}$

\author{
Diffuse MLH1 and PMS2 loss \\ MLH1 promoter methylation»MLH1 germline \\ mutation > MLH1 somatic mutation \\ Diffuse MSH2 and MSH6 loss \\ MSH2 germline mutation > MSH6 germline mutation, \\ EPCAM deletion, MSH2 somatic mutation \\ Diffuse MSH6 loss \\ MSH6 germline mutation > MSH6 somatic mutation \\ Diffuse PMS2 loss \\ PMS2 germline mutation
}

\begin{abstract}
${ }^{\mathrm{a}}$ On rare occasions, the tumor shows loss of expression of three or four proteins together; this may be due to combinations of germline and somatic mutations or germline mutations with MLH1 promoter methylation. Another rare occurrence is loss of protein expression in a geographically discrete portion of the tumor. These cases are thought to have either somatic mutations or MLH1 promoter methylation confined to tumoral subclones.
\end{abstract}
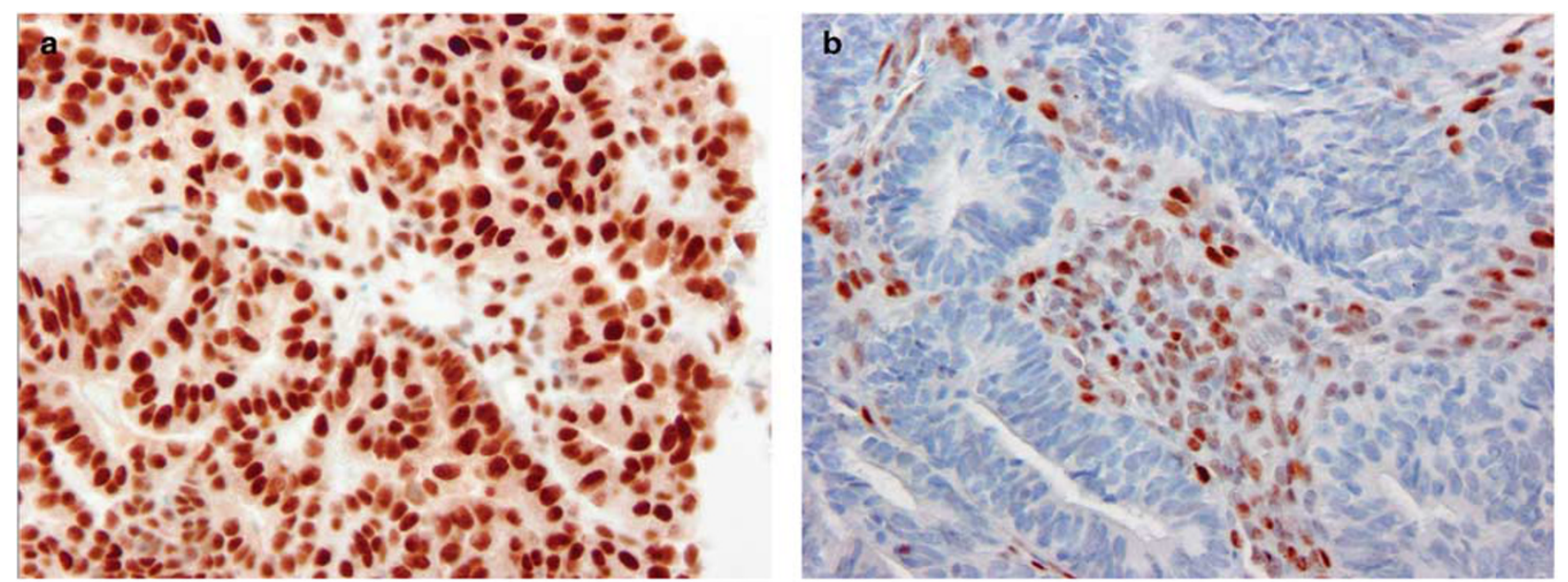

Figure 19 DNA-mismatch repair immunohistochemical staining. Retained nuclear staining for MLH1 in tumor cells (a) and aberrant loss of nuclear staining for MSH2 in tumor cells (b). Note the positive internal control. (This figure was published in Current Concepts in Gynecologic Pathology: Epithelial Tumors of the Gynecologic Tract, An Issue of Surgical Pathology Clinics, 1e, Copyright Elsevier, 2011). 
Table 10 Commonly encountered pitfalls in interpretation of DNA-mismatch repair immunohistochemistry

\begin{tabular}{ll}
\hline Challenge & Solution \\
\hline $\begin{array}{l}\text { Weak or equivocal MLH1 staining } \\
\text { Lacking positive internal control }\end{array}$ & $\begin{array}{l}\text { Use PMS2 staining pattern to adjudicate } \\
\text { Look for proliferative cells, particularly lymphocytes; stain another block if } \\
\text { necessary }\end{array}$ \\
$\begin{array}{l}\text { Anexpected staining patterns (cytoplasmic, nucleolar, } \\
\text { perinuclear, patchy staining) }\end{array}$ & $\begin{array}{l}\text { Any nuclear staining should be scored as 'retained/protein present;' be sure not } \\
\text { to score positive intratumoral lymphocytes alone as 'retained/protein present' }\end{array}$
\end{tabular}

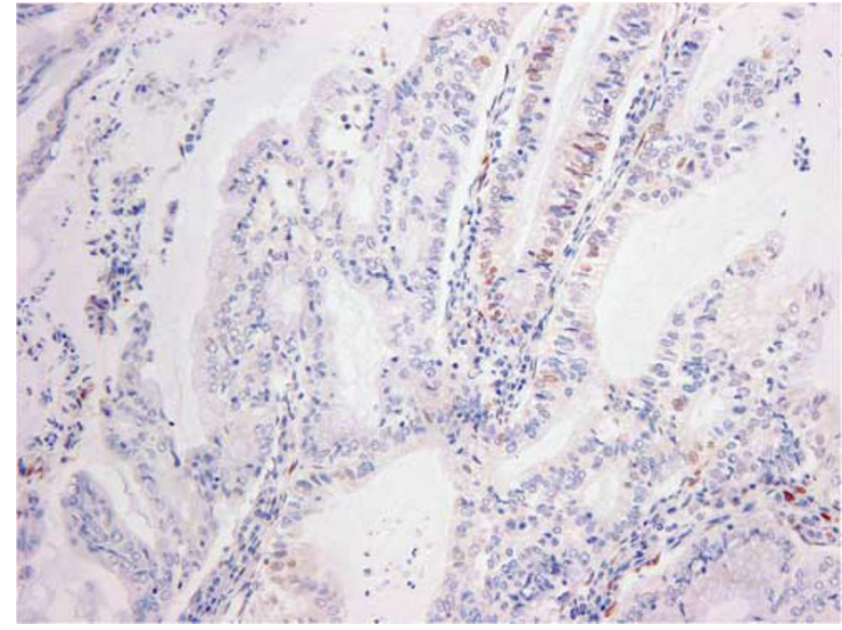

Figure 20 DNA-mismatch repair immunohistochemical staining. Intact but weak staining with MLH1 (shown) can be confirmed with a positive PMS2 immunostain (not shown). (This figure was published in Current Concepts in Gynecologic Pathology: Epithelial Tumors of the Gynecologic Tract, An Issue of Surgical Pathology Clinics, 1e, Copyright Elsevier, 2011).

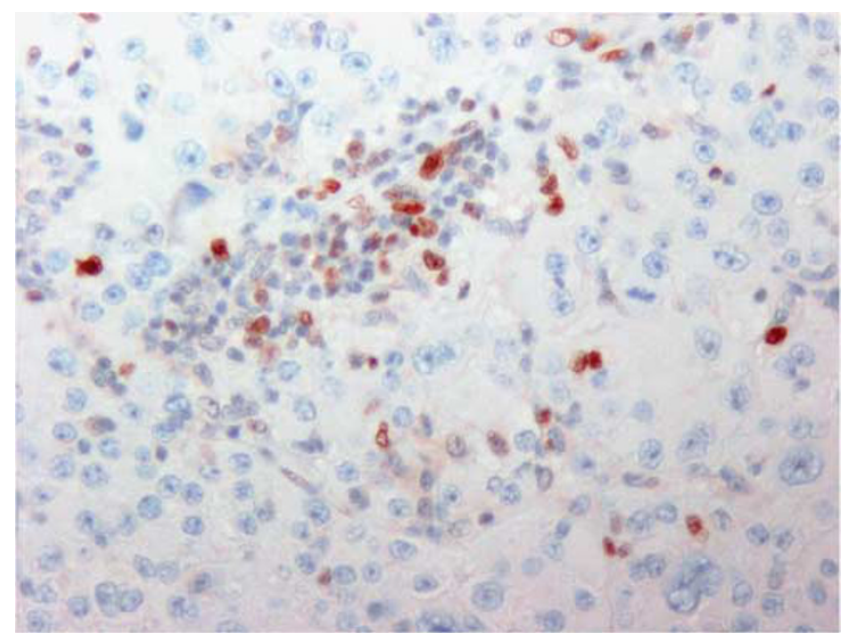

Figure 21 DNA-mismatch repair immunohistochemical staining. Be aware not to confuse intact nuclear staining of lymphocytes as intact nuclear staining of tumor cells. (This figure was published in Current Concepts in Gynecologic Pathology: Epithelial Tumors of the Gynecologic Tract, An Issue of Surgical Pathology Clinics, 1e, Copyright Elsevier, 2011).

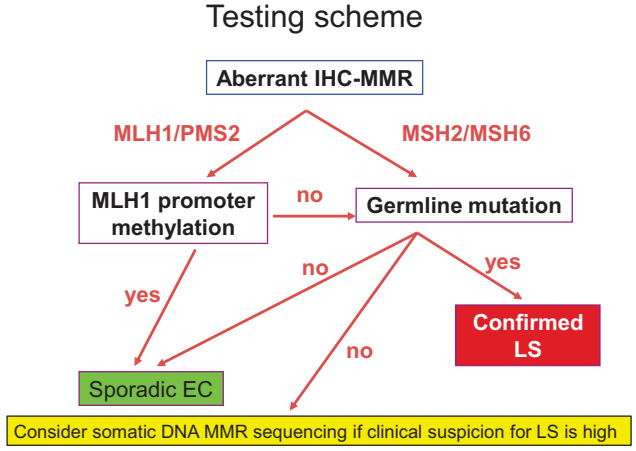

Figure 22 Integrated DNA-mismatch repair immunohistochemical staining and genetic testing schema.

The development of a comprehensive screening system for Lynch syndrome obviously warrants a committed multidisciplinary approach. As we developed our institutional system, we sought advice and collaboration from medical-legal experts, clinical geneticists, gynecologic surgeons, medical oncologists, and pathologists. The appendix found at the end of this article shows a summary of the barriers to the identification of endometrial cancer patients with Lynch syndrome and underscores ways in which even a coordinated and motivated team may miss identifying Lynch syndrome patients. A 'one size fits all' approach to Lynch syndrome screening is most likely not appropriate, but the complexity of any system that is developed requires open lines of communication, compliance audits, and further refinements as practitioners learn more about Lynch syndrome. It is uncertain whether or not this collaborative approach can succeed in low-volume practices and in settings where interdisciplinary teams do not exist or are not highly functional. At a minimum, compliance audits must demonstrate that, regardless of the screening paradigm used, all applicable tumors are tested and all abnormal results are reported to physicians and/or clinical geneticists who can make the best use of the information.

\section{Disclosure/conflict of interest}

The author declares no conflict of interest. 


\section{References}

1 Mutch DG. The new FIGO staging system for cancers of the vulva, cervix, endometrium and sarcomas. Gynecol Oncol 2009;115:325-328.

2 Barlin JN, Soslow RA, Lutz M et al. Redefining stage I endometrial cancer: incorporating histology, a binary grading system, myometrial invasion, and lymph node assessment. Int J Gynecol Cancer 2013;23:1620-1628.

3 Zaino RJ. FIGO staging of endometrial adenocarcinoma: a critical review and proposal. Int J Gynecol Pathol 2009;28:1-9.

4 Hirschowitz L, Nucci M, Zaino RJ. Problematic issues in the staging of endometrial, cervical and vulval carcinomas. Histopathology 2013;62:176-202.

5 Deen S. FIGO staging of endometrial adenocarcinoma: a critical review and proposal. Int J Gynecol Pathol 2009;28:477-478.

6 Ali A, Black D, Soslow RA. Difficulties in assessing the depth of myometrial invasion in endometrial carcinoma. Int J Gynecol Pathol 2007;26:115-123.

7 Jacques SM, Qureshi F, Munkarah A et al. Interinstitutional surgical pathology review in gynecologic oncology: II. Endometrial cancer in hysterectomy specimens. Int J Gynecol Pathol 1998;17:42-45.

8 Kir G, Kir M, Cetiner H et al. Diagnostic problems on frozen section examination of myometrial invasion in patients with endometrial carcinoma with special emphasis on the pitfalls of deep adenomyosis with carcinomatous involvement. Eur J Gynaecol Oncol 2004;25:211-214.

9 Chafe S, Honore L, Pearcey R et al. An analysis of the impact of pathology review in gynecologic cancer. Int J Radiat Oncol Biol Phys 2000;48:1433-1438.

10 Murray SK, Young RH, Scully RE. Unusual epithelial and stromal changes in myoinvasive endometrioid adenocarcinoma: a study of their frequency, associated diagnostic problems, and prognostic significance. Int J Gynecol Pathol 2003;22:324-333.

11 Stewart CJ, Little L. Immunophenotypic features of MELF pattern invasion in endometrial adenocarcinoma: evidence for epithelial-mesenchymal transition. Histopathology 2009;55:91-101.

12 Stewart CJ, Brennan BA, Leung YC et al. MELF pattern invasion in endometrial carcinoma: association with low grade, myoinvasive endometrioid tumours, focal mucinous differentiation and vascular invasion. Pathology 2009;41:454-459.

13 Pavlakis K, Messini I, Vrekoussis T et al. MELF invasion in endometrial cancer as a risk factor for lymph node metastasis. Histopathology 2011;58:966-973.

14 Quick CM, May T, Horowitz NS et al. Low-grade, lowstage endometrioid endometrial adenocarcinoma: a clinicopathologic analysis of 324 cases focusing on frequency and pattern of myoinvasion. Int J Gynecol Pathol 2012;31:337-343.

15 Euscher E, Fox P, Bassett $\mathrm{R}$ et al. The pattern of myometrial invasion as a predictor of lymph node metastasis or extrauterine disease in low-grade endometrial carcinoma. Am J Surg Pathol 2013;37: 1728-1736.

16 Han G, Lim D, Leitao MM Jr et al. Histological features associated with occult lymph node metastasis in FIGO clinical stage I, grade I endometrioid carcinoma. Histopathology 2014;64:389-398.

17 Hertel JD, Huettner PC, Pfeifer JD. Lymphovascular space invasion in microcystic elongated and fragmented (MELF)-pattern well-differentiated endometrioid adenocarcinoma is associated with a higher rate of lymph node metastasis. Int J Gynecol Pathol 2014;33:127-134.

18 Hussein YR, Garg K, Soslow RA et al. Endometrial carcinomas with DNA mismatch repair abnormalities: analysis of 75 cases. Mod Pathol 2013;26:280A.

19 Longacre TA, Hendrickson MR. Diffusely infiltrative endometrial adenocarcinoma: an adenoma malignum pattern of myoinvasion. Am J Surg Pathol 1999;23: 69-78.

20 Logani S, Herdman AV, Little JV et al. Vascular 'pseudo invasion' in laparoscopic hysterectomy specimens: a diagnostic pitfall. Am J Surg Pathol 2008;32:560-565.

21 Kitahara S, Walsh C, Frumovitz M et al. Vascular pseudoinvasion in laparoscopic hysterectomy specimens for endometrial carcinoma: a grossing artifact? Am J Surg Pathol 2009;33:298-303.

22 Folkins AK, Nevadunsky NS, Saleemuddin A et al. Evaluation of vascular space involvement in endometrial adenocarcinomas: laparoscopic vs abdominal hysterectomies. Mod Pathol 2010;23:1073-1079.

23 Krizova A, Clarke BA, Bernardini MQ et al. Histologic artifacts in abdominal, vaginal, laparoscopic, and robotic hysterectomy specimens: a blinded, retrospective review. Am J Surg Pathol 2011;35:115-126.

24 Delair D, Soslow RA, Gardner GJ et al. Tumoral displacement into fallopian tubes in patients undergoing robotically assisted hysterectomy for newly diagnosed endometrial cancer. Int J Gynecol Pathol 2013;32:188-192.

25 McCluggage WG, Hirschowitz L, Wilson GE et al. Significant variation in the assessment of cervical involvement in endometrial carcinoma: an interobserver variation study. Am J Surg Pathol 2011;35: 289-294.

26 Zaino RJ, Abendroth C, Yemelyanova A et al. Endocervical involvement in endometrial adenocarcinoma is not prognostically significant and the pathologic assessment of the pattern of involvement is not reproducible. Gynecol Oncol 2013;128:83-87.

27 Tambouret R, Clement PB, Young RH. Endometrial endometrioid adenocarcinoma with a deceptive pattern of spread to the uterine cervix: a manifestation of stage IIb endometrial carcinoma liable to be misinterpreted as an independent carcinoma or a benign lesion. Am J Surg Pathol 2003;27:1080-1088.

28 Orezzoli JP, Sioletic S, Olawaiye A et al. Stage II endometrioid adenocarcinoma of the endometrium: clinical implications of cervical stromal invasion. Gynecol Oncol 2009;113:316-323.

29 Park KJ, Murali R, Reyes C. Primary cervical carcinoma with secondary adnexal and uterine corpus involvement: detailed morphologic analysis of 14 cases. Mod Pathol 2013;26:291A.

30 Guerra F, Girolimetti G, Perrone AM et al. Mitochondrial DNA genotyping efficiently reveals clonality of synchronous endometrial and ovarian cancers. Mod Pathol 2014;27:1412-1420.

31 Moreno-Bueno G, Gamallo C, Pérez-Gallego L et al. beta-Catenin expression pattern, beta-catenin gene mutations, and microsatellite instability in endometrioid ovarian carcinomas and synchronous endometrial carcinomas. Diagn Mol Pathol 2001;10: 116-122.

32 Kaneki E, Oda Y, Ohishi Y et al. Frequent microsatellite instability in synchronous ovarian and endometrial 
adenocarcinoma and its usefulness for differential diagnosis. Hum Pathol 2004;35:1484-1493.

33 Ulbright TM, Roth LM. Metastatic and independent cancers of the endometrium and ovary: a clinicopathologic study of 34 cases. Hum Pathol 1985;16:28-34.

34 Eifel P, Hendrickson M, Ross J et al. Simultaneous presentation of carcinoma involving the ovary and the uterine corpus. Cancer 1982;50:163-170.

35 Zaino RJ, Unger ER, Whitney C. Synchronous carcinomas of the uterine corpus and ovary. Gynecol Oncol 1984;19:329-335.

36 Montoya F, Martin M, Schneider J et al. Simultaneous appearance of ovarian and endometrial carcinoma: a therapeutic challenge. Eur J Gynaecol Oncol 1989;10: 135-139.

37 Scully RE, Young RH, Clement PB. Tumors of the ovary, maldeveloped gonads, fallopian tube, and broad ligament: Atlas of Tumor Pathology (AFIP Atlas of Tumor Pathology No. 23). American Registry of Pathology. Armed Forces Institute of Pathology: Washington, DC, 1999.

38 Soliman PT, Slomovitz BM, Broaddus RR et al. Synchronous primary cancers of the endometrium and ovary: a single institution review of 84 cases. Gynecol Oncol 2004;94:456-462.

39 Aysal A, Karnezis A, Medhi I et al. Ovarian endometrioid adenocarcinoma: incidence and clinical significance of the morphologic and immunohistochemical markers of mismatch repair protein defects and tumor microsatellite instability. Am J Surg Pathol 2012;36: 163-172.

40 Garg K, Leitao MM Jr, Kauff ND et al. Selection of endometrial carcinomas for DNA mismatch repair protein immunohistochemistry using patient age and tumor morphology enhances detection of mismatch repair abnormalities. Am J Surg Pathol 2009;33: 925-933.

41 Garg K, Soslow RA. Lynch syndrome (hereditary nonpolyposis colorectal cancer) and endometrial carcinoma. J Clin Pathol 2009;62:679-684.

42 Soslow RA, Pirog E, Isacson C. Endometrial intraepithelial carcinoma with associated peritoneal carcinomatosis. Am J Surg Pathol 2000;24:726-732.

43 Baergen RN, Warren CD, Isacson C et al. Early uterine serous carcinoma: clonal origin of extrauterine disease. Int J Gynecol Pathol 2001;20:214-219.

44 Carcangiu ML, Tan LK, Chambers JT. Stage IA uterine serous carcinoma: a study of 13 cases. Am J Surg Pathol 1997;21:1507-1514.

45 Lauchlan SC. Tubal (serous) carcinoma of the endometrium. Arch Pathol Lab Med 1981;105:615-618.

46 Lee KR, Belinson JL. Recurrence in noninvasive endometrial carcinoma. Relationship to uterine papillary serous carcinoma. Am J Surg Pathol 1991;15:965-973.

47 Sherman ME, Sturgeon S, Brinton LA et al. Risk factors and hormone levels in patients with serous and endometrioid uterine carcinomas. Mod Pathol 1997;10: 963-968.

48 Silva EG, Jenkins R. Serous carcinoma in endometrial polyps. Mod Pathol 1990;3:120-128.

49 Spiegel GW. Endometrial carcinoma in situ in postmenopausal women. Am J Surg Pathol 1995;19: 417-432.

50 Euscher ED, Malpica A, Deavers MT et al. Differential expression of WT-1 in serous carcinomas in the peritoneum with or without associated serous carcinoma in endometrial polyps. Am J Surg Pathol 2005;29:1074-1078.
51 Hui P, Kelly M, O'Malley DM et al. Minimal uterine serous carcinoma: a clinicopathological study of 40 cases. Mod Pathol 2005;18:75-82.

52 Yan Z, Hui P. Minimal uterine serous carcinoma with extrauterine tumor of identical morphology: an immunohistochemical study of 13 cases. Appl Immunohistochem Mol Morphol 2010;18:75-79.

53 Jia L, Yuan Z, Wang Y et al. Primary sources of pelvic serous cancer in patients with endometrial intraepithelial carcinoma. Mod Pathol 2015;28:118-127.

54 Jarboe EA, Miron A, Carlson JW et al. Coexisting intraepithelial serous carcinomas of the endometrium and fallopian tube: frequency and potential significance. Int J Gynecol Pathol 2009;28:308-315.

55 McKenney JK, Kong CS, Longacre TA. Endometrial adenocarcinoma associated with subtle lymph-vascular space invasion and lymph node metastasis: a histologic pattern mimicking intravascular and sinusoidal histiocytes. Int J Gynecol Pathol 2005;24:73-78.

56 Frimer M, Khoury-Collado F, Murray MP et al. Micrometastasis of endometrial cancer to sentinel lymph nodes: is it an artifact of uterine manipulation? Gynecol Oncol 2010;119:496-499.

57 Khoury-Collado F, Murray MP, Hensley ML et al. Sentinel lymph node mapping for endometrial cancer improves the detection of metastatic disease to regional lymph nodes. Gynecol Oncol 2011;122:251-254.

58 Cibula D, Abu-Rustum NR, Dusek L et al. Prognostic significance of low volume sentinel lymph node disease in early-stage cervical cancer. Gynecol Oncol 2012;124:496-501.

59 Barlin JN, Khoury-Collado F, Kim CH et al. The importance of applying a sentinel lymph node mapping algorithm in endometrial cancer staging: beyond removal of blue nodes. Gynecol Oncol 2012;125:531-535.

60 Kim CH, Soslow RA, Park KJ et al. Pathologic ultrastaging improves micrometastasis detection in sentinel lymph nodes during endometrial cancer staging. Int J Gynecol Cancer 2013;23:964-970.

61 Abu-Rustum NR. Update on sentinel node mapping in uterine cancer: 10-year experience at Memorial Sloan-Kettering Cancer Center. J Obstet Gynaecol Res 2014;40:327-334.

62 Leitao MM Jr, Kehoe S, Barakat RR et al. Accuracy of preoperative endometrial sampling diagnosis of FIGO grade 1 endometrial adenocarcinoma. Gynecol Oncol 2008;111:244-248.

63 Chi DS, Barakat RR, Palayekar MJ et al. The incidence of pelvic lymph node metastasis by FIGO staging for patients with adequately surgically staged endometrial adenocarcinoma of endometrioid histology. Int J Gynecol Cancer 2008;18:269-273.

64 Mariani A, Dowdy SC, Cliby WA et al. Prospective assessment of lymphatic dissemination in endometrial cancer: a paradigm shift in surgical staging. Gynecol Oncol 2008;109:11-18.

65 Benedetti Panici P, Basile S, Maneschi F et al. Systematic pelvic lymphadenectomy vs no lymphadenectomy in early-stage endometrial carcinoma: randomized clinical trial. J Natl Cancer Inst 2008;100: 1707-1716.

66 ASTEC study group, Kitchener H, Swart AM, Qian Q et al. Efficacy of systematic pelvic lymphadenectomy in endometrial cancer (MRC ASTEC trial): a randomised study. Lancet 2009;373:125-136.

67 Todo Y, Kato H, Kaneuchi M et al. Survival effect of para-aortic lymphadenectomy in endometrial cancer 
(SEPAL study): a retrospective cohort analysis. Lancet 2010;375:1165-1172.

68 Chan JK, Sherman AE, Kapp DS et al. Influence of gynecologic oncologists on the survival of patients with endometrial cancer. J Clin Oncol 2011;29:832-838.

69 Abu-Rustum NR, Iasonos A, Zhou Q et al. Is there a therapeutic impact to regional lymphadenectomy in the surgical treatment of endometrial carcinoma? Am J Obstet Gynecol 2008;198:457.

70 Lutman CV, Havrilesky LJ, Cragun JM et al. Pelvic lymph node count is an important prognostic variable for FIGO stage I and II endometrial carcinoma with high-risk histology. Gynecol Oncol 2006;102:92-97.

71 Barlin JN, Zhou Q St, Clair CM et al. Classification and regression tree (CART) analysis of endometrial carcinoma: Seeing the forest for the trees. Gynecol Oncol 2013;130:452-456.

72 Franchi M, Ghezzi F, Melpignano $\mathrm{M}$ et al. Clinical value of intraoperative gross examination in endometrial cancer. Gynecol Oncol 2000;76:357-361.

73 Altintas A, Cosar E, Vardar MA et al. Intraoperative assessment of depth of myometrial invasion in endometrial carcinoma. Eur J Gynaecol Oncol 1999;20: 329-331.

74 Vorgias G, Hintipas E, Katsoulis M et al. Intraoperative gross examination of myometrial invasion and cervical infiltration in patients with endometrial cancer: decisionmaking accuracy. Gynecol Oncol 2002;85:483-486.

75 Mao Y, Wan X, Chen Y et al. Evaluation of the accuracy of intra-operative gross examination for the surgical management of endometrial cancer. Eur J Obstet Gynecol Reprod Biol 2008;141:179-182.

76 Sato S, Itamochi H, Shimada M et al. Preoperative and intraoperative assessments of depth of myometrial invasion in endometrial cancer. Int J Gynecol Cancer 2009;19:884-887.

77 Mavromatis ID, Antonopoulos CN, Matsoukis IL et al. Validity of intraoperative gross examination of myometrial invasion in patients with endometrial cancer: a meta-analysis. Acta Obstet Gynecol Scand 2012;91: 779-793.

78 Leitao MM Jr, Han G, Lee LX et al. Complex atypical hyperplasia of the uterus: characteristics and prediction of underlying carcinoma risk. Am J Obstet Gynecol 2010;203:349.e1-e6.

79 Leitao MM Jr, Kehoe S, Barakat RR et al. Endometrial sampling diagnosis of FIGO grade 1 endometrial adenocarcinoma with a background of complex atypical hyperplasia and final hysterectomy pathology. Am J Obstet Gynecol 2010;202:278.e1-6.

80 Zikan M, Fischerova D, Pinkavova I et al. A prospective study examining the incidence of asymptomatic and symptomatic lymphoceles following lymphadenectomy in patients with gynecological cancer. Gynecol Oncol 2015;137:291-298.

$81 \mathrm{Lu} \mathrm{KH}$, Dinh M, Kohlmann W et al. Gynecologic cancer as a 'sentinel cancer' for women with hereditary nonpolyposis colorectal cancer syndrome. Obstet Gynecol 2005;105:569-574.

82 Hampel H, de la Chapelle A. The search for unaffected individuals with Lynch syndrome: do the ends justify the means? Cancer Prev Res (Phila) 2011;4:1-5.
83 Järvinen HJ, Aarnio M. Surveillance on mutation carriers of DNA mismatch repair genes. Ann Chir Gynaecol 2000;89:207-210.

84 Pylvänäinen K, Lehtinen T, Kellokumpu I et al. Causes of death of mutation carriers in Finnish Lynch syndrome families. Fam Cancer 2012;11:467-471.

$85 \mathrm{Lu} \mathrm{KH}$, Schorge JO, Rodabaugh KJ et al. Prospective determination of prevalence of lynch syndrome in young women with endometrial cancer. J Clin Oncol 2007;25:5158-5164.

86 Garg K, Shih K, Barakat R et al. Endometrial carcinomas in women aged 40 years and younger: tumors associated with loss of DNA mismatch repair proteins comprise a distinct clinicopathologic subset. Am J Surg Pathol 2009;33:1869-1877.

87 Ramsoekh D, Wagner A, van Leerdam ME et al. Cancer risk in MLH1, MSH2 and MSH6 mutation carriers; different risk profiles may influence clinical management. Hered Cancer Clin Pract 2009;7:17.

88 Win AK, Young JP, Lindor NM et al. Colorectal and other cancer risks for carriers and noncarriers from families with a DNA mismatch repair gene mutation: a prospective cohort study. J Clin Oncol 2012;30: 958-964.

89 Bonadona V, Bonaïti B, Olschwang S et al. French Cancer Genetics Network. Cancer risks associated with germline mutations in MLH1, MSH2, and MSH6 genes in Lynch syndrome. JAMA 2011;305: 2304-2310.

90 Hampel H, Frankel W, Panescu J et al. Screening for Lynch syndrome (hereditary nonpolyposis colorectal cancer) among endometrial cancer patients. Cancer Res 2006;66:7810-7817.

91 Resnick K, Straughn JM Jr, Backes F et al. Lynch syndrome screening strategies among newly diagnosed endometrial cancer patients. Obstet Gynecol 2009;114: 530-536.

92 Modica I, Soslow RA, Black D et al. Utility of immunohistochemistry in predicting microsatellite instability in endometrial carcinoma. Am J Surg Pathol 2007;31:744-751.

93 Mojtahed A, Schrijver I, Ford JM et al. A two-antibody mismatch repair protein immunohistochemistry screening approach for colorectal carcinomas, skin sebaceous tumors, and gynecologic tract carcinomas. Mod Pathol 2011;24:1004-1014.

94 Ryan P, Mulligan AM, Aronson M et al. Comparison of clinical schemas and morphologic features in predicting Lynch syndrome in mutation-positive patients with endometrial cancer encountered in the context of familial gastrointestinal cancer registries. Cancer 2012;118:681-688.

95 Mills AM, Liou S, Ford JM et al. Lynch syndrome screening should be considered for all patients with newly diagnosed endometrial cancer. Am J Surg Pathol 2014;38:1501-1509.

96 Rabban JT, Calkins SM, Karnezis AN et al. Association of tumor morphology with mismatch-repair protein status in older endometrial cancer patients: implications for universal vs selective screening strategies for Lynch syndrome. Am J Surg Pathol 2014;38: 793-800. 


\section{Barriers to the identification of endometrial cancer patients with Lynch syndrome}

1. Problems with patient selection and testing for Lynch syndrome (LS)

a. The rationale for detecting LS in endometrial cancer patients is not as well-delineated as it is in colorectal carcinoma patients. Unlike in colorectal carcinoma, we do not yet know whether LS affects the clinical biology of endometrial cancer or whether therapy should be modulated based on LS status. Therefore, the search for LS may appear mostly as an academic exercise from a gynecologist's standpoint. We emphasize that detecting which endometrial cancer patients have LS saves lives because of the benefit of screening the colonoscopies that lower death rates from any subsequent colorectal carcinomas that develop in the index patient and related carriers, and of prophylactic hysterectomy and bilateral salpingo-oophorectomy in carriers.

b. Many practitioners still use the term 'hereditary non-polyposis colorectal carcinoma syndrome,' which places undue emphasis on colorectal carcinoma. The majority of women with LS develop endometrial and/or ovarian cancer. When they develop both LS-associated endometrial and LS-associated colorectal carcinomas, many patients present with the gynecologic cancer first.

c. Many clinicians depend on a simple family history report to roughly screen for LS. Up to $70 \%$ of endometrial cancer patients with LS do NOT have a personal or family history of LS cancers. This is, again, in contrast to colorectal carcinoma, where rates are in the $20-30 \%$ range.

d. All of this has led to the use of immunohistochemistry for DNA-mismatch repair proteins on tumor samples (IHC for DNA MMR). Centers that do the four-marker panel on all endometrial cancer patients' tumors would theoretically have the lowest miss rates for picking up possible LS cases. This is not a sustainable practice, however.

i. This practice uses many resources, not all of which are reimbursed to pathologists.

ii. This practice mandates the follow-up of an inordinate number of patients who do NOT have LS. Greater than $75 \%$ of patients whose tumors have abnormal DNA MMR IHC have either a somatic DNA-mismatch repair mutation or MLH1 promoter methylation, neither of which constitutes LS. Further testing, given this scenario, requires the complicated, expensive, and time consuming MLH1 methylation assay, which is not available at most medical centers, and in some cases, somatic mutation testing. iii. As MLH1 promoter methylation increases with age, it has been proposed that patients over a certain age should NOT be tested for LS. The incidence of LS does increase with age, but the prevalence falls.

e. Some centers do selective DNA-mismatch repair testing, using patient age, clinical history, tumor morphology, and tumor topography as criteria. Our institutional experience supports continuing our own paradigm, although we acknowledge a number of imperfections. Using a selective approach to testing results in failing to test an occasional LS patient whose age, clinical history, and tumor characteristics do not meet criteria for testing. Another problem is exemplified by a patient whose tumor should have undergone DNA MMR IHC testing according to the selection criteria but was not tested. We currently perform monthly quality assurance checks to catch the latter examples.

f. DNA MMR IHC is $\sim 90 \%$ sensitive, which means that there is always going to be at least a $10 \%$ miss rate if the sole screen is immunohistochemistry, whether performed universally or in a selective manner.

\section{Problems with reporting}

a. The immunohistochemistry report may accompany the main biopsy or hysterectomy report or may be issued as an addendum (issued a few days after the main pathology report). Not finding the report, not finding the report at the right time, and having the wrong person find the report are all barriers. Examples include the following: the clinician does not compulsively check the electronic medical record every day in order to find the addendum report; the report is generated after the patient's first follow-up visit; and the surgeon gets the report, but in many instances, this is not the best person to interpret the report and convey the information to the patient. We have a new quality assurance audit that makes sure that every patient with an abnormal report is contacted by a clinical geneticist. A formal clinical genetics consultation is scheduled only after any necessary ancillary tests are performed (i.e., MLH1 promoter methylation testing) and the patient is motivated to attend the consultation.

b. There are problems with the terminology used in immunohistochemistry reports. Most DNA MMR IHC reports avoid the use of the terms 'LS' and 'germline genetic testing' because then regulators could argue that DNA MMR IHC is a germline genetic test that requires patient consent. The report usually talks about 'lost' or 'retained' expression of DNA-mismatch repair proteins, the subtleties of which may be lost on the recipient. 
3. Problems with clinical genetics consultation and germline genetic testing

a. Many endometrial cancer patients are not motivated to go to their clinical genetics consultation, and there is frequently insufficient encouragement from gynecologists to follow through. This scenario differs from genetic testing of breast cancer and colorectal carcinoma patients. With respect to colorectal carcinoma, most patients need chemotherapy and are aware of the need for multiple different follow-up visits after their surgery. This is different from endometrial cancer in general, where adjuvant therapy is much less frequently needed. In other words, many endometrial carcinoma patients are ready to move on with their lives after hysterectomy.

b. Germline genetic testing cannot be done on tumor material presently, so normal tissue needs to be procured, usually in the form of a blood sample.

c. Once MLH1 promoter methylation is excluded, germline genetic testing detects germline mutations in only approximately $60-70 \%$ of patients with abnormal DNA MMR IHC. Thirty to forty percent have somatic mutations (i.e., not Lynch) or LS, but with germline mutations or other abnormalities that are missed by genetic testing or are incompletely understood. 